\title{
The Role of a Modulatory Neuron in Feeding and Satiation in Aplysia: Effects of Lesioning of the Serotonergic Metacerebral Cells
}

\author{
Steven C. Rosen, ${ }^{1}$ Klaudiusz R. Weiss, ${ }^{1}$ Ronald S. Goldstein, ${ }^{2}$ and Irving Kupfermann ${ }^{1}$ \\ ${ }^{1}$ Center for Neurobiology and Behavior, New York State Psychiatric Institute and College of Physicians and Surgeons of \\ Columbia University, New York, New York 10032, and ${ }^{2}$ The Hebrew University of Jerusalem, Jerusalcm, Isracl
}

\begin{abstract}
Food-induced arousal in Aplysia is characterized by a progressive increase in the speed and strength of biting responses elicited by a seaweed stimulus. Data from semiintact and dissected preparations suggest that the identified, serotonergic, metacerebral cells (MCCs) of the cerebral ganglion contribute to food-induced arousal by enhancing the strength of buccal muscle contractions, and by modulating the output of the central pattern generator for biting movements. In order to test this hypothesis in intact, freemoving animals and to determine if the MCCs play a role in satiation of feeding, the behavior of animals that had their MCCs destroyed by intracellular injection of proteases was compared with that of B Cell-Lesion and Dye Injection control animals (Experiment 1) or surgical control animals (Experiment 2). Nonfeeding behaviors such as defensive withdrawal responses, locomotion, and righting reflexes were unaffected by MCC lesioning. Also unaffected by MCC lesioning were appetitive feeding behaviors and the amount of food needed to satiate animals. Significant behavioral deficits in consummatory feeding behaviors, which remained stable for periods exceeding $10 \mathrm{~d}$, were observed in the MCC-lesioned animals but not in controls. Lesioned animals exhibited a slowing of the rate of repetitive biting responses by $\mathbf{4 0} \%$ of controls and had reduced magnitudes of repetitive bites, particularly at the end of a testing run of 10 consecutive bites. The deficit in bite magnitude was minimally evident in food-deprived animals (Experiment 1) but became more pronounced as animals were fed to satiation (Experiment 2). MCC-lesioned animals still exhibited a residual buildup of the rate and magnitude of biting responses at the onset of feeding behavior. This suggests that, in addition to the MCCs, there are other sources of modulation that contribute to plasticity of consummatory responses during the foodinduced arousal state.
\end{abstract}

Feeding in the mollusc Aplysia is a complex behavior that exhibits many of the characteristics of "motivated" behaviors of higher vertebrates (Kupfermann, 1974a). Two motivational features of feeding in Aplysia that have been investigated are foodinduced arousal and satiation. Food-induced arousal refers to

Received Apr. 22, 1988; revised Sept. 14, 1988; accepted Sept. 19, 1988.

We wish to thank Penny Rosen for technical assistance. Supported in part by PHS Grants MH 35564 and GM 320099.

Correspondence should be addressed to Dr. Irving Kupfermann, Center for Neurobiology and Behavior, Columbia University, New York State Psychiatric Institute, 722 West 168th Street, Annex-Box 25, New York, NY 10032.

Copyright (C) 1989 Society for Neuroscience $0270-6474 / 89 / 051562-17 \$ 02.00 / 0$ a behavioral state that is elicited by food and is expressed in a generalized behavioral activation (e.g., increased heart rate), as well as in specific enhancement of ingestive responses, including an increase in the magnitude and rate of biting responses (Susswein et al., 1978; Weiss et al., 1982). Conversely, satiation of feeding is associated with decreased generalized behavioral activation and a decrease in the magnitude and rate of biting responses (Weiss and Kupfermann, 1977; Susswein et al., 1978; Weiss et al., 1982). Previous investigations of the neuronal systems controlling feeding have suggested that the effects of the food-induced arousal state on the magnitude and speed of biting may be mediated by an identified pair of bilaterally symmetrical neurons that are located in the cerebral ganglion (metacerebral cells, MCCs). These neurons utilize serotonin (5-HT) as their transmitter (Eisenstadt et al., 1973; Weinreich et al., 1973; Gerschenfeld et al., 1978) and have homologs in numerous other molluscs (Weiss and Kupfermann, 1976; Gillette and Davis, 1977; Granzow and Kater, 1977; McCrohan and Benjamin, 1980; Gelperin, 1981; Pentreath et al., 1982; Barber, 1983).

MCC homologs in several molluscan species have been reported to be capable of initiating feeding responses, particularly if fired at high frequency (Gillette and Davis, 1977; Granzow and Kater, 1977; McCrohan and Audesirk, 1987). In Aplysia, on the other hand, although the MCCs innervate the muscles of the organ that executes biting and swallowing (buccal mass), as well as many of the motoneurons that drive these muscles (Cohen et al., 1978; Weiss et al., 1978), firing the MCC at physiological rates does not trigger a feeding motor program, nor does it produce muscle contractions. However, if the MCCs are fired in conjunction with the firing of buccal motoneurons, the force of buccal muscle contractions is enhanced (Weiss et al., 1975, 1978, 1979). Moreover, MCC activity has been shown to increase both the rate of biting responses elicited by food in semi-intact preparations (Weiss et al., 1986b), and the rate of spontaneously occurring buccal motor programs recorded from isolated ganglia (Weiss et al., 1978). These findings, coupled with the observations that the MCCs receive sensory inputs from chemoreceptors and mechanoreceptors with receptive fields on the lips, tentacles, and the buccal mass of the animal (Rosen et al., 1982; Weiss et al., 1986a, b), suggested that the MCCs are one of a class of specialized modulatory neurons that regulate various behavioral expressions of food-induced arousal. Specifically, we suggested that the MCCs modulate the consummatory feeding responses of Aplysia in that MCC activity contributes to the progressive enhancement of the rate and magnitude of biting responses that characterizes the food-induced arousal state.

The preceding hypothesis was originally dependent upon an 
extrapolation of data obtained from semi-intact and isolated ganglionic preparations to the behavior of intact animals. However, the behavior of greatly simplified preparations, particularly for appetitive behaviors such as feeding, is unlikely to be identical to that of intact animals, and in some cases major differences can occur. For this reason, the activity of the MCCs has also been studied in intact, free-moving animals. Recordings of MCC activity in feeding animals has supported the idea that this cell is involved in food-induced arousal since its level of activation correlates with the arousal level of the animal (Kupfermann and Weiss, 1982). In order to test the function of the MCCs in normally feeding animals more directly, we examined the chronic effects of selective, bilateral, single-cell lesioning of the MCCs on the appetitive and consummatory aspects of feeding behavior, as well as on nonfeeding behaviors. The lesioning method used in the present study was modified from that previously reported for destroying individual neurons in the leech (Parnas and Bowling, 1977; Bowling et al., 1978). The method involves the intracellular injection of proteolytic enzymes that diffuse throughout the cell and destroy the soma and cell processes. The injected proteases appear to be ultimately inactivated by autolytic processes within the cell and protease inhibitors outside of the cell, so that damage is confined to the injected neuron.

In the present study we report the results of 2 lesioning experiments. In the first, we studied the chronic effects of MCC lesions on the appetitive and consummatory aspects of feeding in animals that were prevented from swallowing the food. This was done to avoid satiation and thereby minimize variability in the behavior of the animal. In the second experiment, conducted with different groups of animals, we permitted ingestion of the food so that in addition to measures of motor performance, we could determine whether MCC lesioning affects the level at which animals satiate. Our findings confirm, in large measure, predictions derived from a model of the role of the MCCs in food-induced arousal in Aplysia (see Kupfermann and Weiss, 1981; Weiss et al., 1982). MCC lesions did not affect nonfeeding behaviors, appetitive feeding responses, or the amount of food needed to satiate the animals. Behavioral deficits were confined to the rate and magnitude of consummatory biting responses that were measured while animals were not permitted to ingest the test food stimuli. The deficit in bite magnitude, however, was minimally evident in food-deprived animals but became more pronounced as animals were fed to satiation. MCClesioned animals, however, still exhibited a residual build-up of the rate and magnitude of biting responses at the onset of feeding behavior. This finding suggests that, in addition to modulation provided by the MCC, other sources contribute to manifestations of the food-induced arousal state on consummatory responses. Part of the results of the first experiment have been published in the form of a preliminary communication (Rosen et al., 1983).

\section{Materials and Methods}

Subjects. The subjects were Aplysia californica (100-200 gm) provided by the Pacific Bio-Marine Company (Venice, CA). They were maintained in 600 liter tanks of cooled $\left(14-16^{\circ} \mathrm{C}\right)$, aerated, artificial seawater (Instant Ocean, Aquarium Systems Co.) on a 12/12 hr light-dark cycle. The animals were individually housed and tested in perforated plastic cages suspended in the holding tanks.

Experimental groups. In the first experiment, animals were randomly assigned to 1 of 3 experimental groups. A MCC Lesion group $(n=6)$ consisted of animals that had both MCCs surgically destroyed by in- tracellular injections of $1 \%$ protease (Sigma, Type VI) dissolved in an aqueous carrier solution of $50 \mathrm{~mm} \mathrm{KCl}$ with $3 \%$ Fast green dye (see Bowling et al., 1978). A B Cell Lesion control group consisted of animals $(n=7)$ that had at least one large neuron in each of the bilateral $\mathrm{B}$ cell clusters of the cerebral ganglion (Jahan-Parwar and Fredman, 1976) surgically destroyed by injection of protease in carrier solution plus Fast green. B cells are large, easily identified neurons that do not appear to be involved in consummatory phases of feeding. The Dye Injection control group $(n=6)$ consisted of animals that underwent surgical procedures identical to those of animals in the MCC Lesion group except that the control groups' MCCs were intracellularly injected with Fast green in carrier solution less the protease.

In the second experiment, a fresh group of animals was assigned to 1 of 2 groups. The MCC Lesion group $(n=6)$ consisted of animals that had both MCCs destroyed by protease as described above. A surgical control group $(n=6)$ consisted of animals that had surgical procedures identical to those of the MCC Lesion group except that cells in the identified $\mathrm{G}$ cell clusters of the cerebral ganglion (Jahan-Parwar and Fredman, 1976) were impaled, and no materials were injected into the cells. $\mathrm{G}$ cells are large neurons whose cell bodies are close to the MCCs. The control group of Experiment 2 was selected to replace the B Cell Lesion and Dye Injection control groups of Experiment 1 since the postoperative performance of the animals in these groups was found not to differ significantly from preoperative performance, except for the first day following surgery when recovery from surgery affected the performance of all the animals tested.

Surgery. For surgery, animals were immobilized by injection of an isotonic $\mathrm{MgCl}_{2}$ solution (50\% of body weight; Kupfermann, 1974b). They were then secured to a wax-covered dish by means of restraining pins inserted through the anterior tentacles and the tail. The dish was immersed in seawater and inclined at a $40^{\circ}$ angle, so that the animal's hemolymph collected at the tail. A longitudinal incision between the rhinophores was made, and the cerebral ganglion was pinned to an illuminated Lucite stage. The connective tissue sheath on the dorsal surface of the ganglion was thinned, and individual cells were impaled with double-barreled microelectrodes (2-4 $\mu \mathrm{m}$ tip diameter). One barrel contained $2 \mathrm{M} \mathrm{KCl}$ and was used for recording. Solutions (approximately 1-2 nl) were injected from the second barrel at pressures of 5-20 psi. Successful penetrations were determined by the recording of resting and action potentials in the cell. Injections were confirmed by visual observation of the dye filling the cell body. Following the injection, the ganglion was unpinned, the skin was sutured in layers, and the restraining pins were removed. The pins did not produce any obvious damage to the animals and did not appear to interfere with subsequent feeding behavior. Before being returned to its home cage, the animal was allowed $1-2 \mathrm{hr}$ in an isolation chamber in which aerated seawater was frequently exchanged. Postoperative testing began $20-24 \mathrm{hr}$ after surgery.

Procedure. In Experiment 1, animals were fed a single meal of seaweed (Laver, Roland Food Company) upon arrival in the laboratory. Those animals that did not eat were excluded from the experiment, whereas those that did eat were maintained in their home cages for at least a week prior to testing without additional feeding. Behavioral testing then began and consisted of identical preoperative and postoperative daily sessions in which responses associated with the appetitive and consummatory aspects of feeding were measured along with control measures of other, nonfeeding behaviors. One preoperative day of testing (followed immediately by surgery) and 10 postoperative days of testing were administered. The animals received no food throughout the entire period of testing and were not permitted to ingest the food stimuli that were used to elicit feeding responses. Aplysia tolerate long periods of food deprivation with no deleterious effects. Following testing on the 10th postoperative day, the animals were killed and their central ganglia were prepared for electrophysiological and morphological examinations of the lesions.

The behavioral test $\mathrm{cmploycd}$ throughout the expcriment to measurc appetitive and consummatory aspects of feeding is diagrammed in Figure 1 . The experimenter brushed a piece of moistened seaweed $\left(1 \mathrm{~mm}^{2}\right.$, Laver brand) against the tip of one of the animal's rhinophores (Fig. $1 A$, left inset). The animal responded by extending its head towards the stimulus, thereby exposing its lips. As soon as the lips were exposed, the seaweed was brought in contact with the perioral zone and was maintained there while the animal executed a sequence of 10 repetitive biting responses (Fig. 1A, middle inset). Shortly after the first lip touch, animals generally assumed a characteristic feeding posture in which the posterior portion of the foot was fixed to the substrate and the head was 


\section{A}
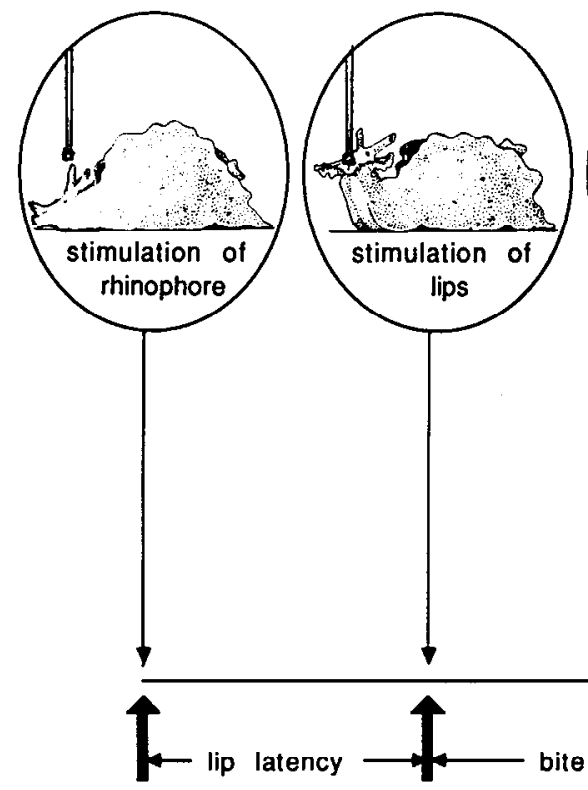

$\checkmark$
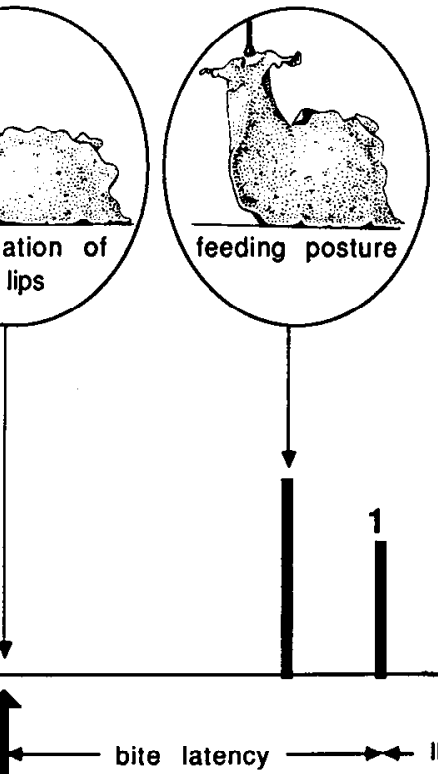

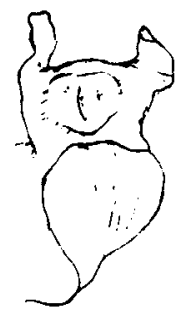

1

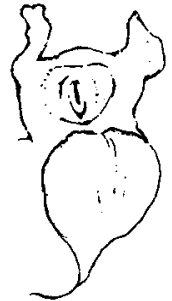

2

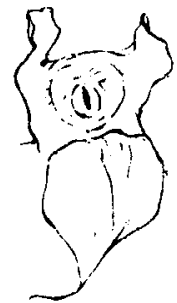

3

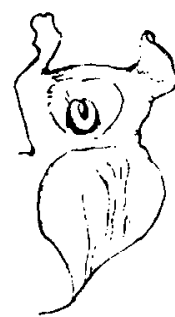

4

\section{BITE NUMBER}
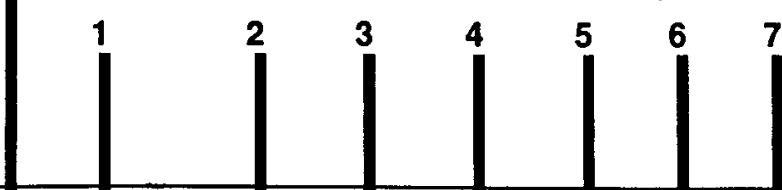

$|B| \# 1-$

Figure 1. Schematic diagram of the stimulus and response events in the behavioral testing procedure. $A$, Typical posture of a quiescent animal (left oval inset), just at the time it is first aroused by gently brushing a $1 \mathrm{~mm}^{2}$ piece of seaweed against its rhinophore; an animal that has been aroused with seaweed to the point where the lips are first exposed (middle oval); and a fully aroused animal that is in the full-up feeding posture (right oval). As soon as the lips were exposed, the seaweed stimulus was applied to the inner portion of the lips and held in place until a series of 10 repetitive biting responses were recorded. The time line shows the sequence of behavioral events in the repetitive biting testing run. The upward arrows indicate the applications of the seaweed stimulus to the rhinophore and lips, respectively. The large vertical line indicates the first appearance of a full-up feeding posture. The following smaller vertical lines (indicated by the numerals $1-10$ ) indicate the repetitive biting responses. The various response measures (lip latency, bite latency, feeding posture latency, and 9 IBIs; see Materials and Methods) are indicated by the horizontal arrows. A bite magnitude score was also determined for each biting response. $B$, Appearance of the lips, mouth, and radula at peak radula forward rotation of 4 separate biting responses. The responses are arranged from left to right in order of increasing bite magnitude as judged by reference to an interval scale of $0-4$. The numbers below each diagrammed response indicate its magnitude: $B 1$, Response characterized by minimal mouth opening; $B 2$, response characterized by mouth opening and the appearance of the radula halves; $B 3$, response consists of mouth opening and radula forward rotation beyond the plane of the mouth opening; $B 4$, response is mouth opening and maximum radula forward rotation through the plane of the mouth so that the muscle attachments of the protracted radula halves are visible. The measures have been shown to have high interobserver reliability (Susswein et al., 1976).

fully raised up to the water surface (Fig. $1 . A$, right inset). Each bite consisted of a mouth opening, an odontophore protraction-retraction sequence (including forward and backward rotation of the radula and opening and closing of the radula halves), and a mouth closing (see Kupfermann, 1974a). As the mouth opened, the experimenter displaced the seaweed to the side to maintain its contact with the lips but prevent its being grasped by the radula. In this manner the experimenter could elicit 10 consecutive biting responses without feeding the animal.

The procedure outlined above permitted the following measures (see Fig. 1A): lip latency, i.e., the time interval between initial touch of seaweed to the rhinophore and exposure of the lips to touch; bite latency, i.e., the time interval between the initial touch of the seaweed to the lips and the peak of the first forward rotation of the odontophore; feeding posture latency, i.e., the time interval between rhinophore touch and appearance of the full-up feeding posture; interbite interval (IBI), i.e., the time interval between successive peaks of odontophore forward rotation; and bite magnitude, the estimate on a scale of $0-4$, of the amplitude of each of the 10 bites elicited by the test stimulus (see Fig. $1 B$ and Susswein et al., 1976). In all, 9 IBIs and 10 bite magnitudes were measured for each testing run.

Three standardized tests (testing runs), outlined above, were administered in a daily testing session. The testing runs were limited to a maximum of $5 \mathrm{~min}$. Fifteen minutes separated the start of each of the successive runs. On rare occasions animals did not execute 10 bites within the $5 \mathrm{~min}$ time limit. Coded entries for missing data were in- corporated into the computer analysis of the statistical results. The effects of missing data were negligible. The righting reflex was tested 5 min after the last run of a daily testing session. For this test, the animal was held upside down above the substrate for $10 \mathrm{sec}$ as it relaxed its body wall muscles. It was then released, and a measurement was made of the time from release to the first walking step which stereotypically follows the torsional righting maneuver. Finally, locomotor responses following the righting reflex, and defensive withdrawal responses to tactile and noxious stimuli were observed and appeared to be normal, although not systematically measured.

In Experiment 2, animals were fed to satiation on a regular basis. They were given one meal every $4 \mathrm{~d}$ for a total of 5 meals. Three (control) meals were fed prior to surgery. The remaining 2 (experimental) meals were fed following surgery. A sixth sham feeding control meal was given to the animals $4 \mathrm{~d}$ following the last experimental meal. On the second day after a meal, repetitive biting responses, swallowing responses, and righting reflexes were measured in animals that were optimally food aroused. The repetitive bite test and the righting reflex test were identical to the behavioral tests in the first experiment. For the swallow test, animals were given strips of seaweed $(1 \times 10 \mathrm{~cm})$, and the time of occurrence of each inward movement of the seaweed was recorded so that a swallow rate could be determined.

The feeding of a meal was arranged so that, in addition to obtaining information about the quantity of food consumed, behavioral measures of bite latency, speed of repetitive biting responses, and bite magnitude 
could be repeatedly made throughout the meal in order to assess the time course of food arousal and satiation in individual animals. This was accomplished by hand feeding the animals uniform pieces of laver, one at a time, and interrupting the procedure after each 10 th piece for a standardized behavioral test. The procedure continued until the rate of responding of the animal declined to a predetermined satiation criterion level. In order to distinguish between cessation of responding due to satiation and pauses in feeding that animals occasionally exhibited, a satiation criterion was defined as 2 consecutive periods of $90 \mathrm{sec}$ each during which a response could not be evoked. The meal was terminated when the criterion was met, either during feeding or during the behavioral test run.

Ten minutes prior to feeding they were removed from their home cages, weighed, and returned to the cages. The feeding procedure began with a behavioral pretest that was similar to the appetitive and consummatory test run of the first experiment. A rhinophore was brushed with a piece of laver until the animal assumed a feeding posture with its head up at the water surface and its lips sufficiently inflated so as to be clearly visible. The test piece of laver was then applied to the medial portion of the lips and continually held in place until a succession of 7 repetitive bites was evoked and recorded. Care was taken to prevent the animal from ingesting the food. On those rare occasions when the animal managed to ingest the food, the test run was aborted and restarted with a fresh piece of laver after a $20 \mathrm{sec}$ delay. Feeding trials were begun immediately following the last test response.

Animals were presented with a fresh bite-size piece of laver $\left(1 \mathrm{~cm}^{2}\right.$ section of a laver sheet; dry weight, $6 \mathrm{mg}$ ). The piece was placed over the mouth of the animal with a forceps and released when it was grasped by the radula. The procedure continued until 10 pieces were consumed. The time between ingestion of one piece and presentation of another was typically several seconds. After the 10 th piece of laver was fed to the animal, feeding was stopped and a behavioral test run, identical to the pretest, was given. The piece was then applied to the perioral zone until 7 repetitive biting responses were again evoked and recorded. Following the 7 th response, feeding trials were resumed until 10 more pieces were ingested and another behavioral test was conducted. Alternate periods of feeding and testing continued until the animal reached satiation.

Sham feeding procedure. On the 4th day following the last experimental meal, a sham control "meal" was fed to each of the MCC Lesion or Control animals. The sham feeding procedure was identical to the normal feeding procedure except that after a biting response was triggered, the laver was withdrawn so that it could not be ingested.

"Blind controls." In order to control for possible experimenter bias, animals were periodically tested by a second experimenter who was unaware of the experimental treatments the animals had received. In Experiment 1 , the second, "blind" experimenter administered 2 additional behavioral testing runs on postoperative testing days 3,6 , and 10 , several hours after testing by the first experimenter had been completed. Since no important differences were observed between the results obtained by the 2 experimenters (see Fig. 3 as an example), the procedure was discontinued in Experiment 2.

Data analysis. The times of occurrence of responses were recorded with a Grass polygraph, and voice recordings were made of the experimenters' estimates of bite magnitudes. In Experiment 2, response times and magnitudes were recorded by means of a custom-designed keyboard and a TRS-80 (model III) microcomputer programmed to store the data on disk and perform a preliminary on-line analysis of individual subject data. Water temperature, testing times, and unusual responses were also indicated. Since the experiments had multiple dependent and independent variables, multivariate analyses of variance were performed with SPSS (Statistical Package for the Social Sciences) computer programs. Since many response measures of Aplysia are highly temperature dependent (unpublished observations), water temperature was included as a covariate to increase the power of the statistical tests.

Histological analysis. Following testing on the 10th postoperative day of Experiment 1, or after the sham feeding control meal of Experiment 2 , an experimenter unfamiliar with the experimental conditions killed the animal, removed the cerebral ganglion with attached nerves and connectives, and examined the condition of its cells under a stereomicroscope. In selected cases, electrophysiological recordings were made of injected and nearby, noninjected cells. The ganglia and connectives were then fixed in $6 \%$ glutaraldehyde and prepared for light and electron microscopy (EM). For EM, the tissues were rinsed, postfixed in $1 \%$ osmium tetroxide, embedded in epoxy resin, and sectioned (see Thompson et al., 1976, for details).
Selection criteria for inclusion of subjects in the experiment. In order to control for variability in the pre- and postoperative health of animals, and for variability in the success of bilateral intracellular injections, a number of strict criteria were used to determine whether animals would be included in the experiment. First, animals were selected from a general laboratory pool if their body weights were in the range of 100 $200 \mathrm{gm}$ and if their mean interbite intervals for the third of a series of repetitive-bite test runs were less than $7.5 \mathrm{sec}$. In our experience, the latter criterion eliminates obviously sick animals but includes the great majority of normal, healthy animals. Second, animals were included only if the surgical treatment to which they were assigned was judged successful. A successful surgery was one in which the initial cell penetrations resulted in the recording of $30-50 \mathrm{mV}$ resting potentials and 40-70 mV spikes; cells were observed to fill evenly with dye upon pressure injection; and immediately following injection, resting and action potentials of the cell did not fall below $50 \%$ of their initial values. Third, animals were included if they recovered from anesthesia and surgical trauma in a normal fashion. Animals were excluded if they remained sensitized to modest tactile stimuli (i.e., withdrew into a spherical defensive posture) for more than one postoperative day or had surgical wounds that opened during the course of behavioral testing. Of the few animals that were rejected for these reasons, no relationship was found to the type of experimental treatment. Finally, animals were rejected after post mortem examination of the ganglia by a "blind" experimenter if evidence was found that the injections failed to fill the cells. Animals with sham injections were included only if the experimenter observed injected cells that were bright green under stereomicroscopic examination. Protease-injected animals were included only if they had bilateral cell remnants characteristic of successful lesions (see Results). Six "lesioned" animals were rejected because of ambiguous post mortem observations, although each had behavioral deficits characteristic of successfully lesioned animals. Three additional animals that were rejected had no behavioral deficits, and at least one MCC was found to be intact. To assure ourselves that our selection procedures were essentially free of bias, we conducted a parallel analysis that included data from animals that were rejected as a result of the post mortem examination. The results of this analysis were in agreement with those of the analysis that excluded animals.

\section{Results}

\section{Morphological confirmation of MCC lesions}

In control animals that had neurons injected with Fast green dye $10 \mathrm{~d}$ previously, the cerebral ganglia were found to contain bright green MCCs that were otherwise normal in appearance and in electrophysiological properties. By contrast, ganglia having neurons injected with protease and dye contained ovoid, mottled, grayish-green cell remnants that appeared to be the injected MCC or B cell somata. The gray-green remnants did not have a negative resting potential or action potentials, although cells immediately bordering were normal both in appearance and in electrophysiological properties. Light and electron microscopic examination of the remnants indicated that they were not neurons but were composed of glia and glial processes (Fig. 2, $A, B$ ). In Aplysia, glia have been shown to be morphologically distinct depending upon their situation in ganglia or in nerves (Coggeshall, 1967; Goldstein et al., 1982). The glia in the region of the cell body remnant exhibited certain typical characteristics. They formed no desmosomes and very rarely contained filaments. In regions of the ganglion away from the site of the protease injection, the glia contained normal lysosome-like structures, approximately $1 \mu \mathrm{m}$ in diameter, of varying electron density. Similar bodies in normal glia found in the interganglionic connectives are smaller and range from 0.3 to $0.5 \mu \mathrm{m}$ in diameter. However, the glia at the site of the lesion were strikingly dissimilar to the glia seen in normal ganglia since they contained, within their cell bodies, enormous lysosomes whose diameters reached 7-8 $\mu \mathrm{m}$, often dwarfing the glial nuclei. The huge lysosomes presumably contained cellular debris from the MCC soma. It is difficult to say at present whether 

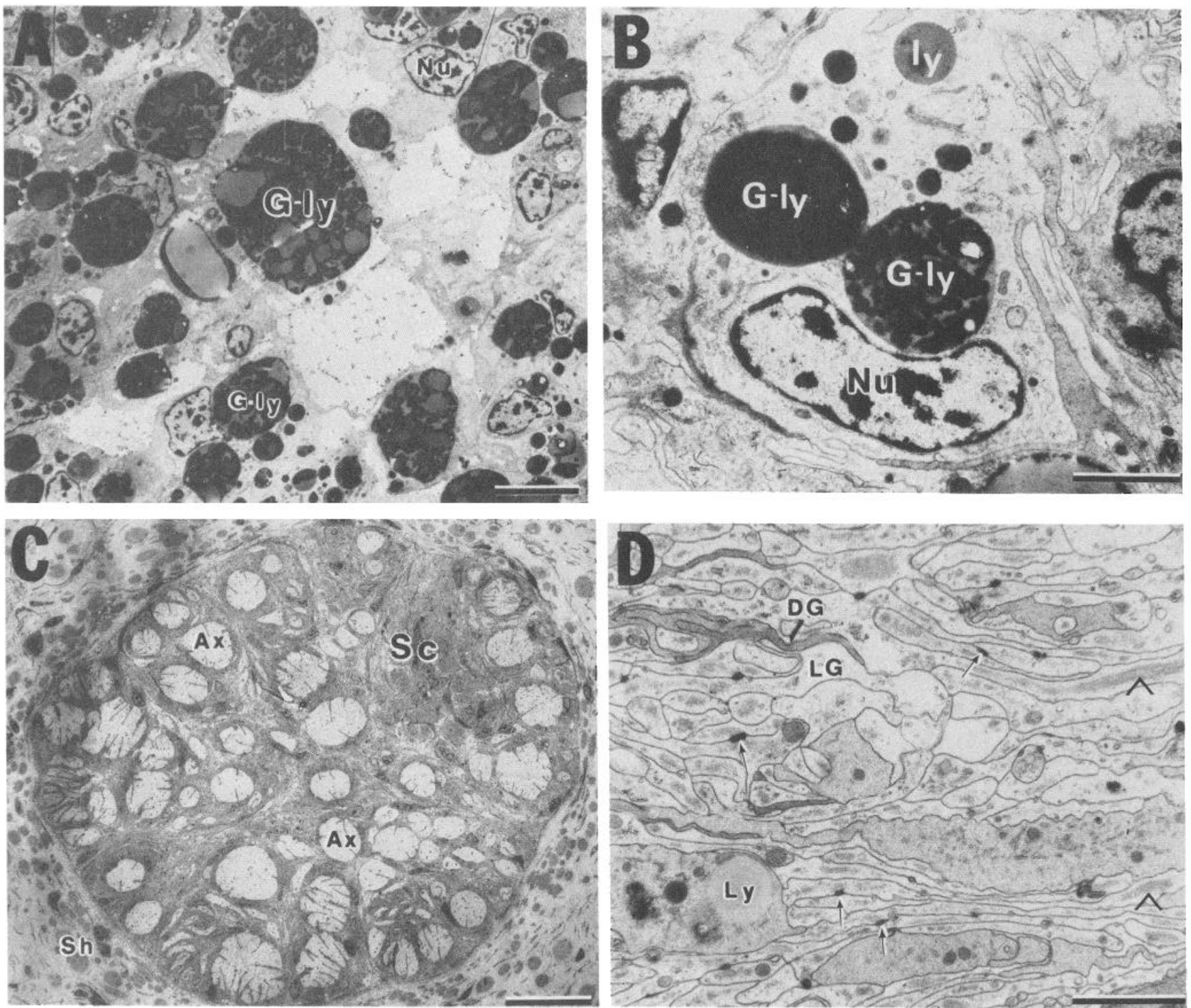

Figure 2. Photomicrographs of the somatic and axonal remnants of lesioned MCCs. A, Low-power electron micrograph of a section of the somatic remnants of a MCC $10 \mathrm{~d}$ after intracellular injection of protease in carrier solution. Numerous glial processes, glial nuclei $(\mathrm{Nu})$, and giant lysosomes contained by the glia $(G-l y)$ are found in the region of the injected cell body. Scale bar, $6 \mu \mathrm{m}$. B, High-power view of a glial cell in the region of the somatic remnants of a lesioned MCC, showing 2 of the giant lysosomes, a glial nucleus, and a lysosome (ly) having dimensions characteristic of those found in the glia of uninjected ganglia. The glia, like those found in normal regions of the ganglia, are characterized by their lack of desmosomes and few filaments, but they are easily distinguished from neurons by their chromatin pattern and nuclear-cytoplasm ratio. Scale bar, $1 \mu \mathrm{m}$. C, Low-power electron micrograph of a transverse section of the C-B connective of an animal whose MCCs were injected with protease in carrier solution $10 \mathrm{~d}$ earlier. The micrograph shows the sheath $(S h)$ surrounding the connective and normal axons $(A x)$ contained in the connective. A scarred region $(S c)$ was found to be devoid of axons following lesioning of the MCCs. Scale bar, $20 \mu$ m. $D$, High-power electron micrograph of the scar shown in $C$. Both light $(L G)$ and dark glial $(D G)$ processes are densely packed in this region, with no axonal cross sections evident. The processes were identified as glial by their longitudinal profiles in this cross-sectional plane, lack of microtubules, extensive filament bundles (caret), and interconnection to one another by desmosomes (arrows). The processes also contain typical lysosome-like structures $(L y)$. Scale bar, $2 \mu \mathrm{m}$.

the glial scar we have observed is primarily due to hypertrophy of the glia, or whether significant glial hyperplasia occurred as well. Mitotic figures were not observed in our tissue samples prepared $10 \mathrm{~d}$ after the lesion, but glial cell divisions may have taken place earlier, following destruction of the neurons.

The cerebral-buccal (C-B) connectives of lesioned and control animals were examined to determine whether MCC cell degeneration proceeded down the axon, towards the terminals of protease injected cells. In light micrographs of transverse sections of the C-B connectives of control animals, the MCC axon was easily recognized, since it was the largest axon and was typically situated in the periphery of the connective. In comparable micrographs of MCC-lesioned animals, an area the size of the MCC axon, but devoid of axons was found in the periphery of the C-B connective (see Fig. 1 in Rosen et al., 1983). EM examination of these areas (Fig. 2, C,D) indicated that they contained whorls of glial processes and glia cell bodies. The whorls of glial processes that filled the space previously occupied by the MCC axon retained the characteristics of glial cell processes found in the nerves. They were attached by numerous desmosomes between their thin processes, and they contained numerous filaments. Montages were made of entire connective cross sections, 
and no other large areas were observed that were devoid of axon profiles and exclusively contained glial cell bodies and glial processes. Furthermore, in many observations of nonlesioned connectives, glial whorls were never noted.

\section{Experiment 1: effects of MCC lesions on feeding} responses in animals not permitted to satiate

\section{Initial analysis of behavioral deficits in biting behavior}

Animals with lesions of the MCCs displayed a marked and consistent deficit in the speed of biting. The degree and consistency of the deficit is shown in Figure 3, in which daily mean IBIs are plotted as a function of successive testing days. On the preoperative day of testing there were no statistically significant differences between the mean IBIs of animals destined to become the MCC Lesion group and control groups. On the first postoperative day of testing all groups showed a marked increase in the IBIs, presumably because of nonspecific effects associated with surgery. Other tests administered on the first postoperative day indicated that the great majority of animals in each of the experimental groups were sensitized to mechanical stimuli and were sluggish in locomotor and orienting responses. Improvements in these behaviors were observed on the second postoperative day of testing when the animals appeared to have fully recovered from the general effects of surgery. On the second postoperative day, all groups showed reductions in their mean IBI scores. The mean scores of B Cell Lesion and Dye Injection control groups returned to their preoperative levels, whereas the scores of the MCC Lesion group dropped to $60 \%$ above preoperative levels. The mean IBI observed for each group on postoperative day 2 persisted unchanged for the remaining $8 \mathrm{~d}$ of testing. The performance of the MCC Lesion group, although significantly deficient compared with that of the control groups

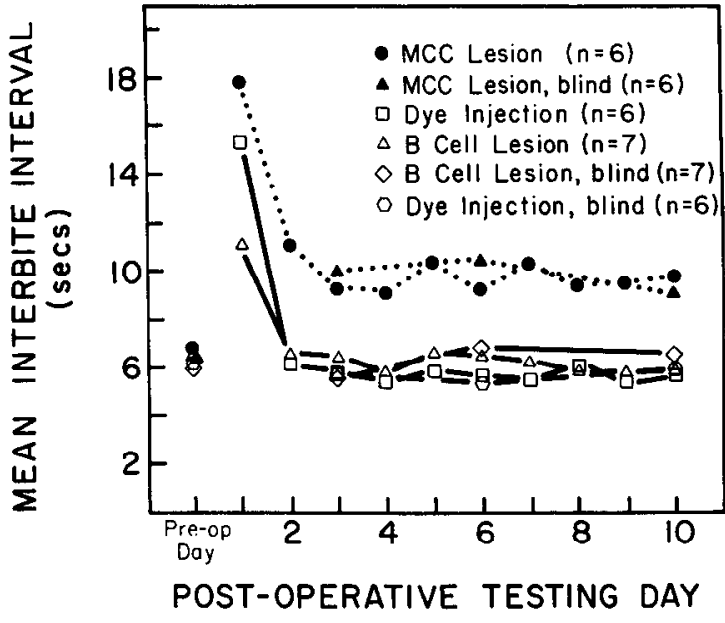

Figure 3. MCC Lesion, B Cell Lesion, and Dye Injection control group mean interbite intervals (IBIs) plotted as a function of the 10 successive postoperativc days of testing. The leftmost points represent group means for the preoperative day of testing (Pre-op Day). Three additional curves show the group data collected by the second (blind) experimenter on the preoperative testing day and on postoperative lesting days 3,6 , and 10. The "blind" experimenter had no knowledge of the animals' group assignments and prior histories. For the sake of clarity, SEMs have been omitted from the curves.

and with its own preoperative performance was nonetheless stable and showed neither signs of recovery of function nor further deterioration with time.

Figure 3 also shows the results of the measurements of IBI made independently by the second ("blind") experimenter who had no knowledge of the group assignments and treatments of

Table 1. Summaries of four-way repeated measures analysis of variance results for first and second ("blind") experimenter interbite interval measurements

\begin{tabular}{|c|c|c|c|c|c|c|}
\hline & \multicolumn{3}{|c|}{ First experimenter } & \multicolumn{3}{|c|}{ Second experimenter } \\
\hline & $d f$ & $\mathrm{~F}$ & Signif. & $d f$ & $\mathrm{~F}$ & Signif. \\
\hline \multicolumn{7}{|l|}{ Between subjects } \\
\hline A (Lesion) & 2 & 37.01 & $0.001^{a}$ & 2 & 10.73 & $0.004^{a}$ \\
\hline Subj. w. groups & 15 & & & 15 & & \\
\hline \multicolumn{7}{|l|}{ Within subjects } \\
\hline B (Days) & 2 & 0.72 & 0.493 & 2 & 0.97 & 0.418 \\
\hline $\mathrm{AB}$ & & 1.48 & 0.234 & & 0.43 & 0.784 \\
\hline \multicolumn{7}{|c|}{ B $\times$ subj. w. groups } \\
\hline C (Runs) & 1 & 13.92 & $0.002^{a}$ & 1 & 0.23 & 0.164 \\
\hline $\mathrm{AC}$ & & 0.44 & 0.644 & & 0.02 & 0.983 \\
\hline \multicolumn{7}{|c|}{$\mathrm{C} \times$ subj. w. groups } \\
\hline $\mathrm{D}$ (Bites) & 5 & 7.06 & $0.001^{a}$ & 5 & 5.11 & $0.001^{a}$ \\
\hline $\mathrm{AD}$ & & 6.07 & $0.001^{a}$ & & 3.37 & $0.002^{a}$ \\
\hline \multicolumn{7}{|c|}{$\mathrm{D} \times$ subj. w. groups } \\
\hline $\mathrm{BC}$ & & 1.74 & 0.192 & & 1.36 & 0.283 \\
\hline $\mathrm{ABC}$ & & 2.24 & 0.088 & & 0.43 & 0.782 \\
\hline $\mathrm{BD}$ & & 1.21 & 0.287 & & 0.54 & 0.855 \\
\hline $\mathrm{ABD}$ & & 1.40 & 0.131 & & 1.50 & 0.098 \\
\hline $\mathrm{CD}$ & & 1.53 & 0.192 & & 3.50 & $0.009^{a}$ \\
\hline $\mathrm{ACD}$ & & 1.78 & 0.077 & & 1.04 & 0.430 \\
\hline $\mathrm{BCD}$ & & 1.01 & 0.434 & & 0.82 & 0.613 \\
\hline$A B C D$ & & 2.09 & $0.007^{a}$ & & 0.82 & 0.680 \\
\hline
\end{tabular}

\footnotetext{
${ }^{a}$ Significant at the 0.01 level.
} 


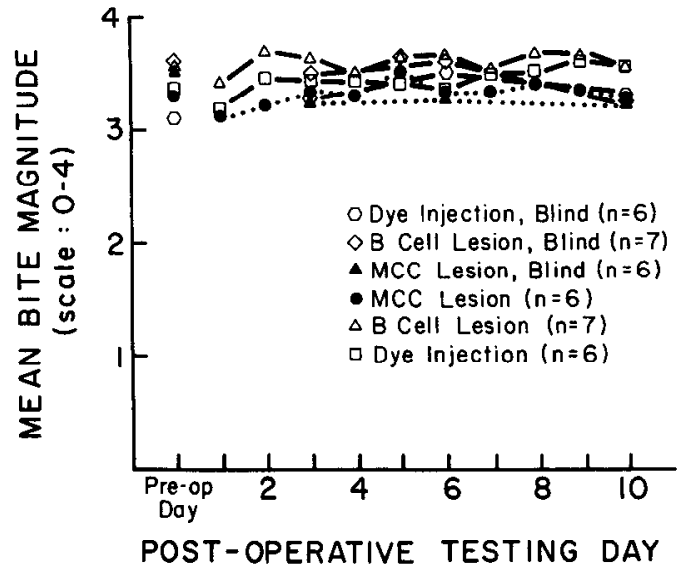

Figure 4. MCC Lesion, B Cell Lesion, and Dye Injection control group mean bite magnitude scores plotted as a function of the 10 successive postoperative days of testing. The leftmost points represent group means for the preoperative day of testing (Pre-op Day). The graph also shows the measurements made by the "blind" experimenter on the preoperative day of testing and on postoperative testing days 3,6 , and 10 . No statistically significant group differences were found. SEMs have been omitted for the sake of clarity.

the animals. The "blind" experimenter administered 2 additional testing runs to each of the experimental animals on the preoperative day of testing and on postoperative days 3, 6, and 10. His findings closely parallel those of the first experimenter. Repeated measures, analysis of variance tests made with the IBI data collected by each experimenter showed the same pattern of statistically significant differences between the experimental and control groups (Table 1), suggesting that experi- menter bias had a negligible effect on the behavioral measurements.

Whilc an analysis of overall mean bite magnitudes for the various groups over days (Fig. 4, Table 2) failed to reveal the significant lesion deficits indicated by the analysis of interresponse measures, a finer-grained evaluation (reported below) showed an effect of MCC lesions on bite magnitude that was obscured by grouping all of the responses within runs in the computation of overall group means.

Our initial analysis provided the basis for further analyses of the data. To summarize, the initial data indicated that (1) the animals were generally debilitated on the first postoperative day of testing; (2) subsequent postoperative performance of each of the experimental groups was stable over days; and (3) there was no significant interobserver difference in response measurement. For these reasons our subsequent analyses excluded postoperative day 1 data, combined the data for postoperative days 2-10, and focused on the data collected by the first experimenter, since these were most complete.

\section{Specificity of the deficits}

In order to assess the specificity of the behavioral deficit resulting from MCC lesions, we computed group mean difference scores (postoperative minus preoperative scores for each animal) for selected appetitive and consummatory feeding behaviors and for a nonfeeding behavior (righting timc). These results are summarized in Figure 5 which is a series of bar graphs showing group difference scores (postoperative overall means of days 210 minus means of preoperative scores) for behavioral measures, including feeding posture latency (a measure of the appetitive phase of feeding, Fig. $5 A$ ); IBI and bite magnitude (2

Table 2. Summaries of four-way repeated measures analysis of variance results for first and second ("blind") experimenter bite magnitude measurements

\begin{tabular}{|c|c|c|c|c|c|c|}
\hline & \multicolumn{3}{|c|}{ First experimenter } & \multicolumn{3}{|c|}{ Second experimenter } \\
\hline & $d f$ & $\mathbf{F}$ & Signif. & $d f$ & $\mathrm{~F}$ & Signif. \\
\hline \multicolumn{7}{|l|}{ Between Subjects } \\
\hline A (Lesion) & 2 & 0.98 & 0.396 & 2 & 0.00 & 0.998 \\
\hline Subj. w. groups & 15 & & & 15 & & \\
\hline \multicolumn{7}{|l|}{ Within Subjects } \\
\hline B (Days) & 2 & 0.11 & 0.893 & 2 & 2.50 & 0.113 \\
\hline $\mathrm{AB}$ & & 0.16 & 0.958 & & 0.44 & 0.777 \\
\hline \multicolumn{7}{|c|}{$B \times$ subj. w. groups } \\
\hline $\mathrm{C}$ (Runs) & 1 & 17.03 & $0.001^{a}$ & 1 & 6.81 & 0.128 \\
\hline $\mathrm{AC}$ & 2 & 1.01 & 0.388 & 2 & 0.54 & 0.600 \\
\hline \multicolumn{7}{|c|}{$\mathrm{C} \times$ subj. w. groups } \\
\hline D (Bites) & 5 & 37.14 & $0.001^{a}$ & 5 & 18.24 & $0.001^{a}$ \\
\hline $\mathrm{AD}$ & & 0.91 & 0.532 & & 0.56 & 0.840 \\
\hline \multicolumn{7}{|c|}{$\mathrm{D} \times$ subj. w. groups } \\
\hline $\mathrm{BC}$ & & 0.72 & 0.496 & & 0.63 & 0.543 \\
\hline $\mathrm{ABC}$ & & 5.04 & $0.003^{\circ}$ & & 0.66 & 0.626 \\
\hline $\mathrm{BD}$ & & 0.53 & 0.865 & & 1.05 & 0.411 \\
\hline $\mathrm{ABD}$ & & 1.55 & 0.074 & & 1.33 & 0.181 \\
\hline $\mathrm{CD}$ & & 2.50 & $0.039^{b}$ & & 4.61 & $0.002^{a}$ \\
\hline $\mathrm{ACD}$ & & 0.90 & 0.895 & & 0.93 & 0.514 \\
\hline $\mathrm{BCD}$ & & 0.91 & 0.533 & & 1.27 & 0.260 \\
\hline $\mathrm{ABCD}$ & & 1.59 & 0.063 & & 1.01 & 0.456 \\
\hline
\end{tabular}

${ }^{a}$ Significant at the 0.01 level.

"Significant at the 0.05 level. 


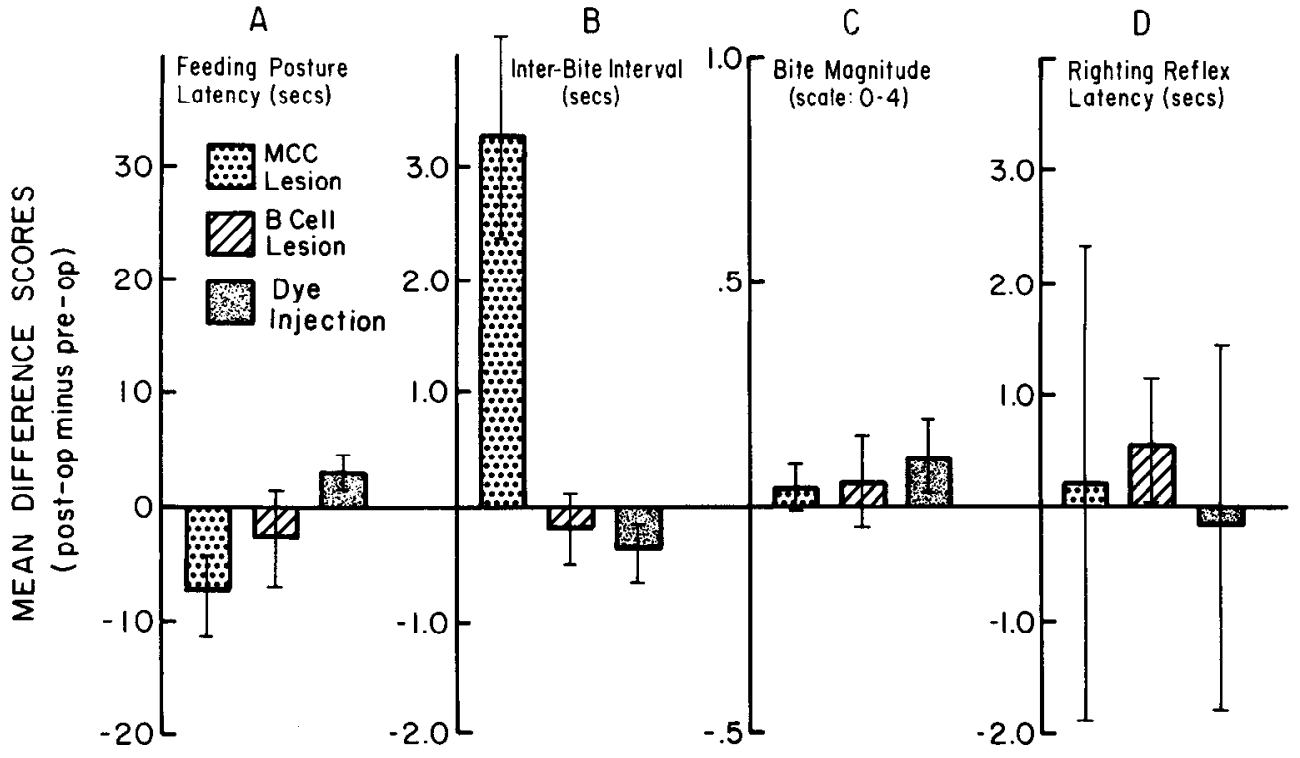

Figure 5. MCC Lesion $(n=6)$, B Cell Lesion $(n=7)$, and Dye Injection control $(n=6)$ group mean difference scores (postoperative overall mean of days 2 10 minus the mean of preoperative scores, \pm SEMs) for various behavioral measures: $A$, The time to exhibit the full feeding posture; $B$, interbite interval; $C$, bite magnitude; and $D$, righting reflex latency. With the exception of bite magnitude (expressed in arbitrary units of $0-4$, with $0=$ smallest magnitude), the ordinate scales are in seconds. measures of the consummatory phase of feeding, Fig. 5, $B, C$ ); and righting reflex latency (a measure of a nonfeeding response, Fig. $5 D$ ). Only the difference scores for the IBI measure showed group differences that were statistically significant (see analysis of variance, Table 1). The MCC Lesion group IBI difference score was significantly greater than comparable scores for the B Cell Lesion or the Dye Injection control groups.

\section{Characteristics of the deficit resulting from MCC lesions}

The slowing of the biting responses of MCC-lesioned animals was examined in greater detail by an analysis of videotape recordings made for selected animals during the first 7 biting responses of a testing run. The biting responses were subjected to a standardized, frame-by-frame video analysis in which measurements were made of the width of mouth opening (expressed as a percentage of maximum). During radula movements, the radula remains in close contact with the mouth, and as the radula rotates forward it appears to push open the mouth. Therefore, width of mouth opening was found to be a reliable indicator of the extent of radula forward rotation and could also be used as a reliable measure of the overall duration of the radula forwardbackward rotation sequence. This analysis revealed several ways in which the behavior of MCC-lesioned animals differed from their preoperative behavior and from that of B Cell-lesioned controls. First, as expected, the overall frequency of repetitive bites was lower for the MCC-lesioned animals (Fig. 6, $A, B 2$ ). Second, compared with controls (Fig. 6BI), the duration of mouth opening (protraction-retraction) for each biting cycle was exceptionally long relative to the portion of the cycle when the mouth was closed (Fig. 6, $A, B 2$ ). The alteration of the cycle was particularly evident for the first bites in a testing run (see in particular Fig. 6A). Third, the prolonged duration of the radula protraction-retraction sequence appeared to be related more to retarded backward rotation than to an actual slowing of the forward rotation phase of the biting sequence since the rising phases of the biting cycle curves for the MCC-lesioned animals were as steep as those of controls, whereas the falling phases were delayed for the MCC-lesioned animals. Frequently, the duration of the radula protraction-retraction sequence for the first bites of a daily session was so long that it appeared that the radulas of the MCC-lesioned animals were stuck in the maximally protracted position. Figure $6 A$ shows an example of an MCC-lesioned animal exhibiting a marked "stuck" radula. Five of the six MCC-lesioned animals, but none of the 13 controls, exhibited marked prolongation of radula forward rotation ( $p<0.01$, Fisher test). Figure $6 B 1$ shows a typical example of the mouth opening responses (radula protractions) seen in all normal animals, while Figure $6 B 2$ shows the mouth opening responses of the same animal after bilateral MCC lesions.

\section{Modulation of biting responses in MCC-lesioned animals: analysis across successive biting responses}

Although the data presented thus far indicate that MCC-lesioned animals have a pronounced and stable deficit in the speed of their repetitive biting responses, the video analysis suggested that the animals may retain a surprising capacity for modulation of their consummatory responses. This capacity is best illustrated when mean IBIs are computed for each of the successive IBIs within a testing run. The group mean IBI measures (displayed in Fig. 7) for successive bites within a run represent grand means for the 3 daily testing runs conducted for postoperative days $2-10$. As seen in Figure 7 , both the initial mean bite latency (leftmost data point) and the first mean IBI of the MCC Lesion group were $100 \%$ longer than the means for the B Cell Lesion and Dye Injection control groups. The mean IBIs of the MCC Lesion group declined markedly over the succeeding 4 bites and reached a minimum that was approximately $30 \%$ greater than control. Subsequently, the means slowly rose again for the remaining bites within a run. In this experiment, the control groups had very short initial latencies, indicating that they were maximally aroused, perhaps because of persisting food stimuli in the seawater. Consequently, they showed no subsequent change of IBI.

A second component of the modulation of consummatory responses in MCC-lesioned animals was seen when mean bite magnitude measures were plotted for successive bites within a testing run (Fig. 8). Postoperatively, each of the experimental groups showed a small but consistent build-up in bite magnitude during the first 4 bites within a testing run. The build-up occurred within a range of approximately a half of a unit of bite 
Figure 6. Temporal sequence of mouth openings and closings during 7 repetitive biting responses elicited in $2 \mathrm{MCC}$ lesioned animals. The sequence was reconstructed from a frame-by-frame analysis of videotaped biting responses. Width of mouth opening is expressed as a percentage of the maximum opening observed for the individual animal for a given run. $A$, Responses of an MCC-lesioned animal showing a marked "stuck" radula phenomenon. $B$, Responses of a normal animal $(B I)$ and those of the same animal following bilateral MCC lesioning ( $B 2)$.

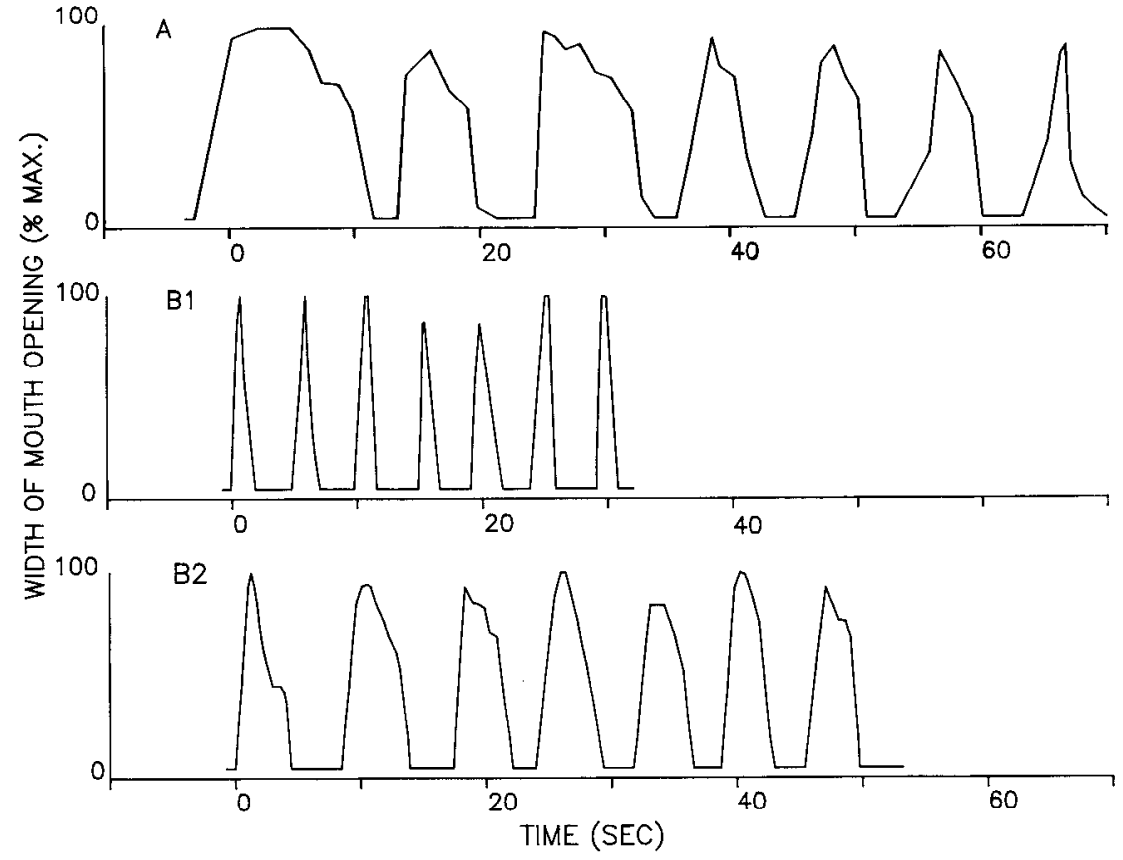

Table 2 presents the results of the overall multivariate statistical analysis with bite magnitude as the main dependent variable. As seen in Table 2, neither the data collected by the first or second experimenter showed a significant effect of Lesion (factor A) or Days (factor B) on bite magnitude. Both sets of data, however, did show a significant Bites (factor D) effect, and bites by run by experimental group complex interaction effect.

\section{Effects of MCC lesions on successive bite magnitudes within a testing run: analysis of preop-postop difference scores}

In order to compensate for intersubject variability, and thereby enhance the sensitivity of our measure of bite magnitude, we analyzed the data in terms of difference scores. For each successive bite within-a-run, for each animal, we subtracted the mean preoperative bite magnitude measurement from the mean postoperative measurement, and then computed group means. Bite magnitude difference scores for each of the successive bites constituting single testing runs revealed a markedly different pattern for MCC-lesioned animals compared with the control groups (Fig. 9). The B Cell Lesion control and Dye Injection control groups showed negligible difference scores, with little systematic change over the 10 successive bites within-a-run, and therefore the control curve represents their pooled data. In contrast to the controls, the bite magnitude difference scores of the MCC-lesioned animals, were positive and high for the first 3 bites at the beginning of the testing run, and then became increasingly negative for the bites at the end of the run. These data indicate that for the first 3 bites of the testing run, on the average, the bite magnitudes of the MCC-lesioned animals were larger than they were before the lesion (i.e., mean difference scores are greater than zero). For the last 7 bites, the MCClesioned animals showed smaller bite magnitudes compared with their preoperative scores (i.e., mean difference scores are less than zero). A repeated-measures MANOVA revealed a significant group by bite interaction $[\mathrm{F}(2,9)=2.14, p<0.01]$, suggesting that the change in bite magnitude difference scores over 


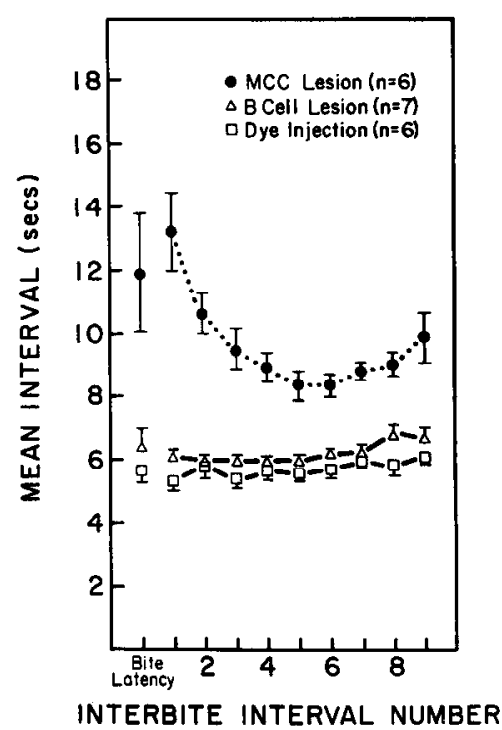

Figure 7. MCC Lesion, B Cell Lesion, and Dye Injection control group mean interbite intervals ( \pm SEMs) plotted as a function of the interbite interval number within the testing run. The leftmost points represent mean bite latencies for each of the experimental groups. Succeeding points represent interbite intervals. Each group mean is based on 3 daily measurements obtained for each animal of a group (one for each of the 3 testing runs administered within a day) averaged across postoperative testing days $2-10$.

successive bites within a run is different for the lesion and control groups.

\section{Experiment 2: effects of MCC lesions on feeding to satiation}

The results of the experiment reported in the following section indicate that, although bilateral lesioning of the serotonergic MCCs affects biting behavior, it does not grossly disrupt the ability of the animal to ingest a normal-sized meal. Moreover, in these experiments we show that the effect of the lesions on bite magnitude is correlated with the level of satiation of the animal. Differences between lesioned and control animals were found to increase gradually during the latter halves of the feeding of complete meals.

\section{Effects of MCC lesions on body weight, meal weight, and rate of feeding}

Figure 10 (right) shows average body weights for the MCC Lesion and control groups of animals measured immediately prior to each of the 3 preoperative control meals, the 2 postoperative meals, and the postoperative sham meal (meal number 6). No significant differences were found between groups, either before and after surgery, nor were postoperative body weights within groups significantly different from the preoperative weights. Overall, the groups showed a slight increase in body weight throughout the entire experiment, presumably due to normal growth.

In contrast to the modest weight gains by animals in the MCC Lesion and control groups throughout the experiment, the meal sizes of the animals showed relatively large increases, particularly during the preoperative control period of feeding. Figure 10 (left) shows the average meal sizes of the MCC lesion and Control groups for each of the pre- and postoperative meals. The wet weight of laver eaten by a subject was divided by the

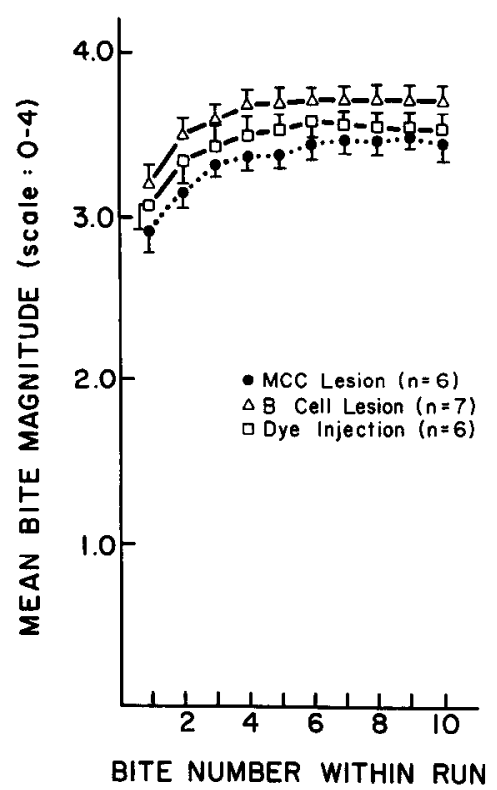

Figure 8. MCC Lesion, B Cell Lesion, and Dye Injection control group mean bite magnitudes ( \pm SEMs) plotted as a function of bite number within a testing run. Each group mean is based on 3 daily measurements obtained for each animal of a group (one for each of the 3 testing runs administered within-a-day) averaged across postoperative testing days 2-10.

body weight of the animal prior to the meal and the quotient was expressed as a percentage. Both groups showed a comparable increase in meal size over the 3 preoperative meals. Moreover, the meal size of both groups continued to increase slightly for the first postoperative meal and remained approximately at that level for the second. The relatively small size of the first meal of the experiment may be due to the fact that the animals

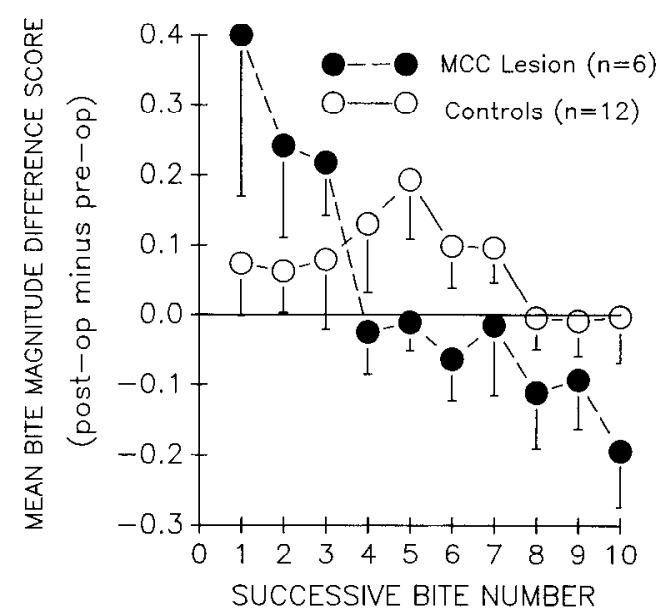

Figure 9. MCC Lesion $(n=6)$ and Control group $(n=12)$ mean bite magnitude difference scores (postoperative minus preoperative) plotted for successive bite number within a run. The control data represents the pooling of data for the B-Cell Lesion and Dye Injection control animals. For each successive bite within-a-run, for each animal, the mean preoperative bite magnitude score was subtracted from the mean postoperative bite magnitude score, and then group means were computed. Each postoperative group mean includes data from the 3 daily testing runs administered on postoperative testing days 2-10. Each preoperative group mean is based on the 3 daily testing runs for the single preoperative day of testing. Vertical lines represent SEMs. 

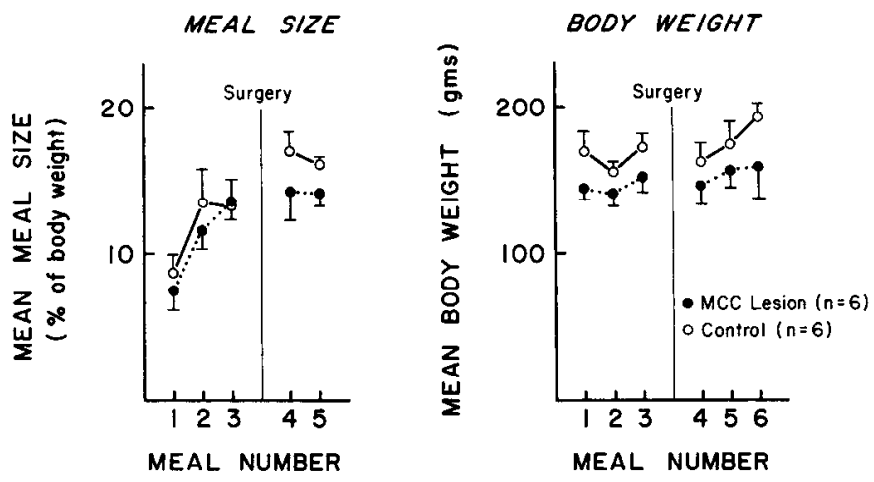

Figure 10. Mean meal sizes (left graph) and mean body weights (right graph) for the MCC Lesion $(n=6)$ and Control $(n=6)$ groups of animals measured for preoperative meals $(1-3)$ and postoperative meals (4-6). For each meal of each animal, meal size (expressed as percentage of body weight) was computed by dividing the wet weight of the laver eaten during the meal by the animal's body weight measured just prior to the meal. Since the last postoperative meal was a sham feeding control meal, no meal size data were obtained. Vertical lines represent SEMs.

may not have been fully acclimated to laver as a food source at the beginning of the experiment. Alternatively, the period of starvation prior to the experiment may have diminished their appetites (see Hamilton, 1969). To compensate for these possibilities, the feeding data for the first preoperative meal were eliminated from the subsequent analysis. In order to summarize the data so that appropriate comparisons could be made to determine whether MCC lesioning affected meal size, a combined preopcrative feeding mean and a combined postoperative mean were computed for each animal. The combined means were the averages of meal sizes for the 2 preoperative meals, prior to surgery, and the 2 postoperative meals following surgery. The combined scores ( \pm the SEMs) for each group were next computed. Tests between pairs of combined means failed to show any significant differences between lesioned and control animals for pre- or postlesion meal size. Moreover, within each group, correlated $t$ tests failed to show significant differences between preoperative versus postoperative combined mean meal size.

Since the first experiment showed that animals with $\mathrm{MCC}$ lesions exhibited slower than normal rates of repetitive biting when ingestion of the food was prevented, measurements of meal durations and of bite rates were made to determine whether the impaired rate of repetitive biting (tested while food was not ingestcd) translated into slower feeding during the actual meal. No significant group differences were observed in the duration of any of the experimental meals. To determine if the repetitive IBI was increased in the MCC-lesioned animals, as was found in Experiment 1, we examined the mean IBIs of Control and MCC-lesioned animals, tested by continuous exposure to a piece of food (without ingestion of the food) following every 10 pieces eaten during the meals. The data confirmed the findings of the first experiment: the mean IBIs of the MCC Lesion group on the days of the 2 postoperative meals were significantly greater than the IBIs of the control group (see Fig. 11 and subsequent section). Moreover, the postoperative IBIs of the MCC Lesion group were longer than the group's own IBIs for the last 2 preoperative meals.

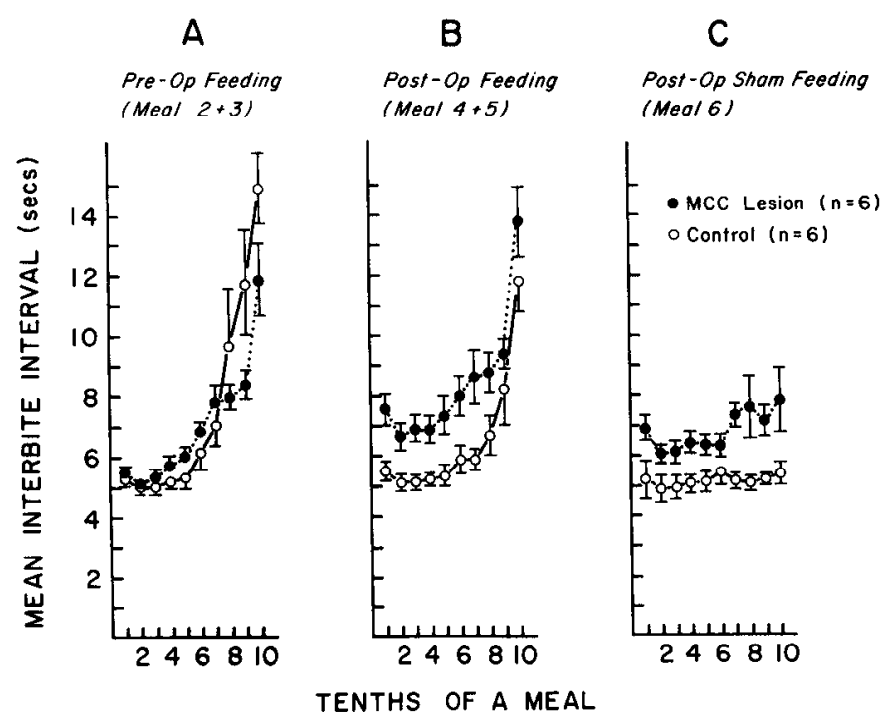

Figure 11. Mean interbite intervals of MCC Lesion and Control groups of animals plotted as a function of successive 10ths of a meal. $A$, Combined results for the 2 meals immediately prior to surgery. $B$, Combined results for the 2 meals following surgery. $C$, Results of the single postoperative sham feeding control meal that followed the preceding meals. For each animal whose data contributed to the curves shown in $A$ and $B$, respectively, a combined score for each 10 th of a meal was first determined. The combined score was based on the mean interbite intervals computed for the individual test runs given during a particular 10 th of a meal averaged for that 10 th of a meal across the 2 combined meals. The group means of the subjects' combined scores \pm SEMs are shown.

\section{Interaction of the effects of $M C C$ lesioning and satiation on consummatory response parameters}

In order to study the interaction of the effects of MCC lesioning and the process of satiation in the groups of experimental animals, it was necessary to transform (Vincentize) the data of individual subjects to compensate for the fact that meal size was highly variable, both between the subjects and for successive meals eaten by the same subject. Thus, each meal of each animal was divided equally into tenths (deciles) based on the total quantity of laver consumed by the animal. The number of test runs (without ingestion) that occurred within each decile was then determined, and average bite latencies, IBIs, and bite magnitudes were calculated for each subject. For each decile of each meal, group means for bite latency, successive IBI, and successive bite magnitude were then computed.

\section{Effects of MCC lesioning on mean IBIs over successive meal deciles}

When the mean IBIs of MCC Lesioned and control groups (measured within noningestion testing runs) were plotted for successive meal deciles, a pattern of results emerged that is indicative of a consistent deficit of MCC-lesioned animals in the speed of biting, similar to that seen in Experiment 1 . The plots of group mean IBIs (tested without ingestion) as functions of meal decile are shown in Figure 11. Figure $11 \mathrm{~A}$ shows the results for each group when data collected for the 2 meals immediately prior to surgery were combined; Figure $11 R$ shows group means when the 2 postoperative meals were combined; and Figure $11 \mathrm{C}$ shows results for the single postoperative sham feeding meal. Preoperatively, both groups showed similar overlapping functions that were not significantly different. At the 
start of a meal, IBIs averaged about $5 \mathrm{sec}$. A slight reduction was seen in the mean IBI for the second decile compared with the first, presumably the result of food arousal. Then, as a result of satiation, both groups showed a slowly accelerating increase in IBI.

Postoperatively, a distinct difference was seen in the group mean IBI functions. Whereas the postoperative IBI function of control animals was no different from its preoperative function, the postoperative IBI function for the MCC Lesion group differed from both the group's own preoperative function and the Control group's postoperative function. A MANOVA for the data showed a significant group by day interaction $[F(2,9)=$ $7.45, p<0.05]$. The postoperative group differences are apparent for the first, and for all subsequent, meal deciles (Fig. 11B). Although there was an approximately $40 \%$ increase in the mean IBIs of the MCC Lesion group, the shape of the curve for the MCC-lesioned animals was quite similar to, and appears to parallel, the Control group curve. The IBIs for deciles 2-5 were less than that of the first decile. Then, during deciles 5-8 the IBIs gradually increased. Sharper increases were seen for deciles 9 and 10 . Figure $11 \mathrm{C}$ shows the results for the postoperative sham feeding. Again, the mean IBIs of the MCC Lesion group were significantly greater than the Control group for all deciles. Both groups showed a slight decline in mean IBI after the first decile. Whereas the Control group means remained unchanged during the course of the sham meal, a small but consistent increase was observed in the MCC Lesion group IBIs for the last 4 meal deciles. The latter finding suggests that animals with bilateral MCC lesions may be more susceptible to response decrement due to fatigue or sensory adaptation associated with prolonged food stimulation and repetitive biting.

\section{Effects of MCC lesions on mean bite magnitudes over successive meal deciles}

When bite magnitudes (measured during noningestion testing runs) were averaged for the MCC Lesion and Control groups and plotted for successive meal deciles (Fig. 12), functions that appear to be reciprocals of those for mean IBIs were observed. Figure $12 A$ shows preoperative group data in which the results of the 2 meals prior to surgery are combined; Figure $11 B$ shows group data when the 2 postoperative meals were combined; Figure $11 C$ shows the results for the sham feeding control meal. Preoperatively, for the first 4 deciles, the bite magnitudes for both groups of animals were near maximum (approximately 3.5 out of a possible maximum of 4.0 ). At the 5 th decile, due to satiation, bite magnitudes started to decline in a decelerating fashion, so that by the 10 th decile they reached their lowest values, approximately $60 \%$ of maximum. Preoperatively, only small and statistically nonsignificant differences existed between groups.

Postoperatively, a different pattern of results for bite magnitude was seen (Fig. 12B). The shapes of the functions were the same for MCC Lesion and Control animals, i.e., a high magnitude value for the first decile was followed by near maximum values for deciles $2-4$, and then decelerating values for bite magnitudes for the subsequent deciles. For all deciles, the mean bite magnitudes for the MCC Lesion group were less than for controls. For the first half of the meal the MCC Lesion group's bite magnitudes were only slightly less than those of the control, but during the second half of the meal (deciles 6-10), the scores of the 2 groups diverged. The bite magnitudes of the MCC Lesion group became smaller than that of the Control

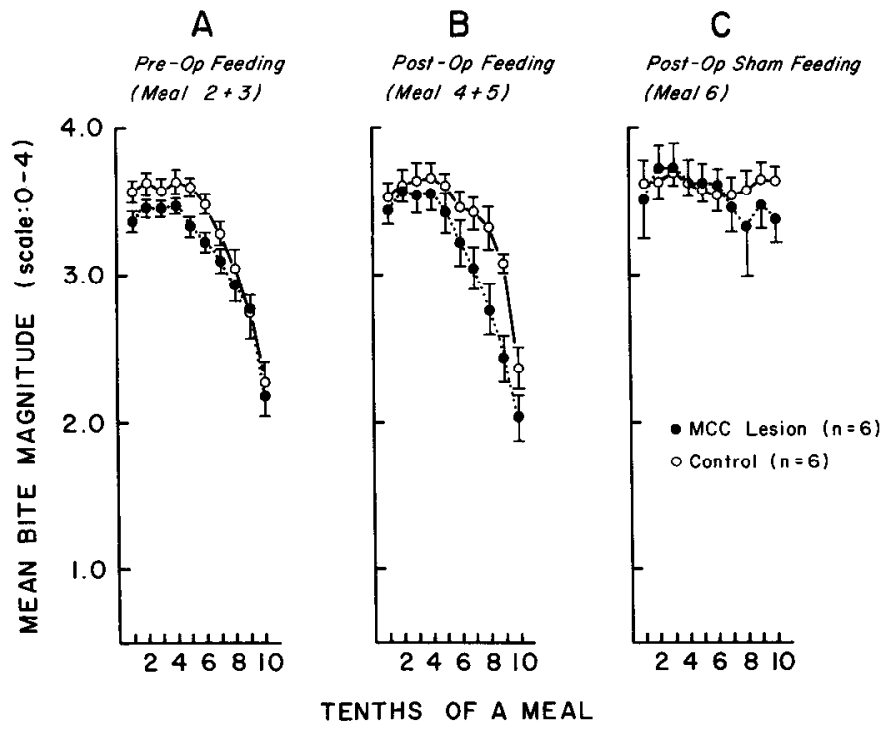

Figure 12. Mean bite magnitudes ( \pm SEMs) of MCC Lesion and Control groups of animals plotted as a function of successive 10ths of a meal. $A$, Combined results for the 2 meals fed immediately prior to surgery. $B$, Combined results for the 2 meals following surgery. $C, \operatorname{Re}-$ sults of the single postoperative sham feeding control meal that followed the preceding meals. For each animal whose bite magnitude data contributed to the curves shown in $A$ and $B$, respectively, a combined score for each 10 th of a meal was first determined. The combincd score was based on the mean bite magnitude scores computed for the individual test runs given during a particular 10th of a meal averaged for that 10th of a meal across the 2 combined meals.

group. Small postoperative group differences were also consistently seen in the bite magnitude data for the sham feeding control meal. Mean bite magnitudes for both groups were nearly identical for the first 6 meal deciles, showing no decrease in maximal bite magnitude. During the last 4 meal deciles the MCC Lesion group showed a slight but statistically insignificant decline in bite magnitude scores, which may reflect the susceptibility of MCC-lesioned animals to the fatiguing effects of prolonged repetitive biting responses. No changes across deciles were seen during sham feeding in the control group.

In order to reveal more clearly the possible group differences in bite magnitude in relation to meal decile, we computed for each animal a series of mean bite magnitude difference scores by subtracting preoperative mean bite magnitudes for each decile from postoperative bite magnitudes for corresponding deciles. The group mean difference scores for successive deciles are shown in Figure 13. The resulting difference score functions indicate that for both the MCC Lesion and the control group, significant postoperative differences in bite magnitude scores were found for the last 3 meal deciles but not for any of the preceding deciles, although for the first 5 meal deciles the MCC Lesion group consistently had small positive difference scores. The negative values in the difference scores of the MCC Lesion group for the last 3 meal deciles are indicative of a postoperative decrease of the magnitude of biting responses. On the other hand, the positive values in the difference scores of the control group for the last 3 deciles are indicative of an apparent increase in the magnitude of biting responses following surgery. A repeated-measures MANOVA for bite magnitude differences indicated a significant group by decile interaction $[F(1,9)=5.25$, $p<0.01]$. 


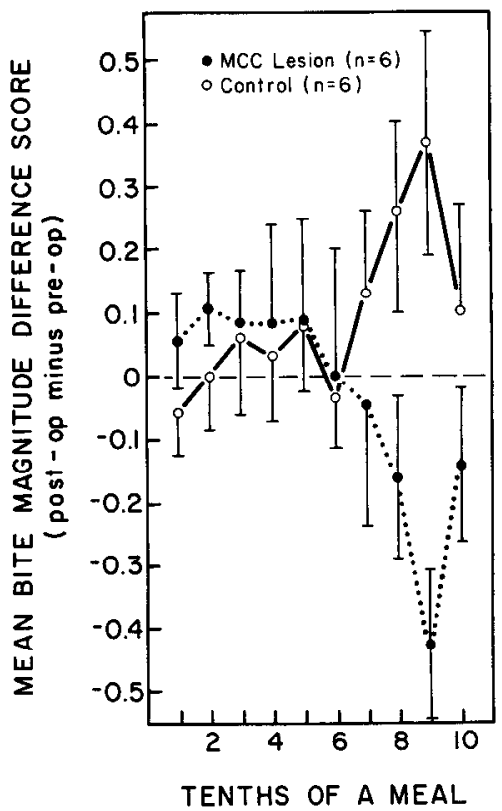

Figure 13. MCC Lesion and Control group mean ( \pm SEMs) bite magnitude difference scores (postoperative minus preoperative) plotted as a function of successive 10ths of a meal. For each 10th of a meal eaten by each animal a mean bite magnitude score was computed, and a combined bite magnitude decile score for the 2 meals prior to surgery and the 2 meals following surgery was then calculated. Finally, postoperative minus preoperative bite magnitude differences for corresponding decile scores were computed.

As a mcans of cvaluating the statistical significance of our experimental analysis of possible interactive effects of $\mathrm{MCC}$ lesions and the process of satiation, we performed 3-way multivariate analyses of variance tests on mean data for our IBI, bite magnitude, and bite latency scores. In the repeated measures design, subjects were nested under experimental group. The other factors were day (combined preoperative days versus combined pustoperative scores) and decile. For the analysis of IBI scores there was a significant decile effect $[F(1,9)=43.3, p<$ $0.001]$, as expected. The analysis also indicated significant interaction effects of group and decile $[F(1,2,9)=2.44, p<0.05]$ and group and day $[F(1,2,9)=4.25, p<0.01)$. These findings indicate that the deficits in consummatory feeding that result from lesioning the MCCs can, in turn, be modified by changes in the state of the animal (e.g., arousal) that occur during the process of satiation.

\section{Effect of MCC lesions on swallowing}

Previous data have shown that the repetitive swallowing response observed when a strip of seaweed is ingested represents a consummatory behavior clearly distinct from the repetitive biting response observed when animals are stimulated with food, but are not permitted to ingest the food (Chiel et al., 1986; Kupfermann, 1974a; Weiss et al., 1986b). Comparisons of mean interswallow intervals on the test when animals were presented with a strip of seaweed, failed to reveal any postoperative group differences. The mean swallow rate for controls was 4.04 swallows $/ \mathrm{min}( \pm 0.38 \mathrm{SEM})$ and that for the MCC-lesioned animals was 3.82 swallows $/$ min $( \pm 0.30$ SEM). Moreover, no significant differences were found between the pre- and postoperative performance of each group (Fig. 14). All groups showed a characteristic increase of interswallow interval during the first 3

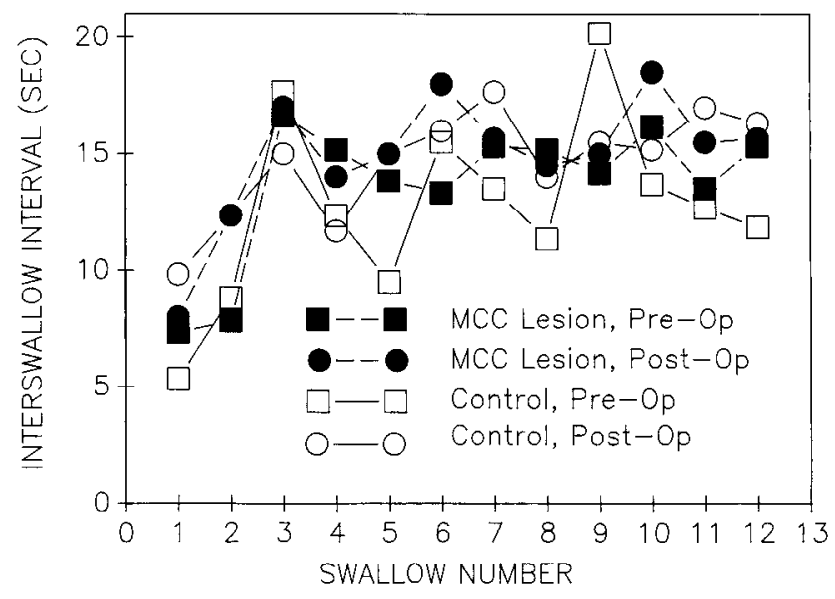

Figure 14. MCC Lesion and Control group mean interswallow intervals measured during ingestion of strips of seaweed. Strip tests were administered to animals $2 \mathrm{~d}$ following each of the pre- and postoperative meals. Group means for the first 12 swallows of combined preoperative and combined postoperative tests are shown. No significant group differences were found. For the sake of clarity SEMs have been omitted.

swallows, followed by a decrease, and then subsequent oscillations over the remaining swallows. It should be noted that the interswallow interval showed great variation, and therefore a small effect of the lesion could be obscured.

\section{Discussion}

\section{Efficacy of the chronic single-cell lesioning technique}

The results of the present experiments indicate that the technique for destroying single cells in the intact animal by intracellular injection of proteolytic enzymes is an effective means for the selective and complete removal of identified neurons in Aplysia. The protease-injected MCC somata were completely destroyed, while there was no discernible morphological damage or physiological dysfunction in neighboring cells in the central ganglia. Moreover, the data indicate that the destruction extends down a main axonal branch of the MCC. Although we have at present no direct morphological evidence that the proteases destroy the terminals of the MCC, we expect, based on the analysis of the efficacy of the technique in the leech and cockroach (Parnas and Bowling, 1977; Bowling et al., 1978; Comer, 1985) that this is indeed the case. Our finding that the lesions result in a specific behavioral deficit, which remains stable over the course of several weeks, provides support for the conclusion that there was complete destruction of the cell. We conclude that single cells in the molluscan CNS can be effectively eliminated by protease injection, both in a morphological and functional sense. Furthermore, the behavioral deficits we have observed are in large measure very likely the result of cell elimination and not the result of other processes related to surgical trauma or diffuse actions of proteases, since neither our Dye Injection control group or B Cell Lesion control group exhibited behavioral abnormalities. We cannot completely rule out the possibility that the effects we observed may be due to sprouting or other changes of nonlesioned neurons in response to the $\mathrm{MCC}$ lesioning or damage of surrounding tissue (see, for example, Altrup and Kolde, 1982; Bulloch and Kater, 1982; Murphy et al., 1985; Allison and Benjamin, 1986). Arguing against this possibility are the relatively rapid development (within $24 \mathrm{hr}$ ) and the great stability (over $10 \mathrm{~d}$ ) of the observed deficits. 
The technique utilized in this study permits analysis of singlecell function by the traditional lesion method and has enabled us to test various hypotheses concerning cell function in the unanesthetized, free-moving animal. This type of study is particularly important for the investigation of cells that contribute to the control or modulation of a complex behavior, such as feeding, that involves appetitive and consummatory components that may not be completely normal in semi-intact or dissected preparations. As is the case for all lesion techniques (Chow, 1967), singlc-ccll lcsions do not dircctly establish the function of the lesioned tissue, but instead determine the capacities of the cells remaining after the lesion. The possibility of inferring function from a lesion is greatly enhanced when this technique is combined with a theoretical approach that makes specific predictions about the effects of lesions. Our own experiments were guided by a specific model of the functional role of the MCCs in the food-induced arousal system of Aplysia (Kupfermann and Weiss, 1981; Weiss et al., 1982). The behavioral deficits we have observed support many features of this model.

\section{Postulated role of the MCCs in food-induced arousal}

Our model of food-induced arousal postulates that arousal is mediated by a multifaceted system comprised of a central arousal component that controls the persistent, or mnemonic, aspects of arousal and an executive component that produces specific behavioral effects. The central component receives inputs from a variety of sensory modalities and distributes excitatory or inhibitory inputs to the executive subsystems that mediate specific arousal effects appropriate for each of the behaviors within the behavioral repertoire of the animal. The MCCs are thought to comprise part of one of the executive subsystems that mediates the effects of arousal on feeding. Within this subsystem it was hypothesized that MCC actions are restricted to the consummatory, but not to the appetitive, aspects of feeding. Consummatory feeding behaviors are thought to be under the control of a central pattern generator (see Susswein and Byrne, 1988) that functions to orchestrate neuronal output for coordinated feeding movements. The pattern generator, or the command system driving the generator (Rosen et al., 1987), receives input from the central arousal system and provides output to the motoneurons that innervate the biting apparatus (buccal mass). The MCC is postulated to be extrinsic to the motor system per se. It provides neuromodulatory input to the consummatory feeding system at many levels, including the effector level (muscle), as well as the motoneuronal and pattern-generating levels. Previous data, based on semi-intact preparations or isolated ganglia, suggested that $\mathrm{MCC}$ input to buccal central pattern generators increases the cycle rate of one or more buccal ganglion programs (Weiss et al., 1978, 1986a; sec also, Gelperin, 1981). Our finding that chronic lesion of the MCC slows the rate of biting is consistent with these data.

\section{Lack of effect of MCC lesioning on the amount of food needed to reach satiation}

The model of the functional role of the MCCs does not specifically predict what the effects of MCC lesioning should be on satiation of feeding. Satiation in Aplysia is due to inhibitory feedback from the gut and is expressed by changes in both appetitive and consummatory responses (Susswein and Kupfermann, 1975a, b; Kuslansky et al., 1987). The activity and responsiveness of the MCCs gradually decreases as animals satiate (Kupfermann and Weiss, 1982). This suggests an interest- ing possible mechanism of satiation: Cessation of feeding could be due to depression of MCC activity, which, in turn, leads to a weakening of biting responses to the point where no responses can occur. The results of the experiments reported in this paper, however, argue against this mechanism since the satiation level appeared to be unaffected by MCC lesions. Although MCC lesioning did not affect satiation as measured by the total amount of food that animals ate, lesioning did alter the relationship between bite magnitude and level of satiation (Fig. 12). Both MCC-lesioncd and nonlesioncd animals cxhibited a decrease of bite magnitude over the course of the meal, but for the lesioned animals, the decrease was relatively greater towards the end of the meal.

\section{$M C C$ lesioning effects are limited to the consummatory phase of feeding}

The model of the role of the MCCs in the expression of foodinduced arousal makes a number of predictions regarding the performance of animals following selective removal of the MCCs. First, neither nonfeeding behaviors nor appetitive feeding behaviors should be affected by the lesions, i.e., the effects of the lesions should be confined to the consummatory aspects of feeding. Second, within the domain of consummatory responses, the lesions should not affect the initiation or sequencing of the feeding responses but should affect the rate and strength of the rcsponses. The bchavior of MCC-lesioncd animals conforms in large measure to these predictions. The findings that locomotor activity, defensive responses, and righting reflexes are all normal following $\mathrm{MCC}$ lesioning indicate that the effect of the lesioning is specific to feeding. The findings that head waving, orientation to a food source, and time to exhibit a characteristic feeding posture are also normal indicate that the effect of the lesioning is specific to the consummatory, but not the appetitive, phase of feeding. The data showing that the lesioning results in a consistent increase in bite latency and a slowing of repetitive biting indicate that the MCCs are, indeed, involved in a consummatory phase of feeding. Since animals can still ingest food following MCC lesioning, the MCCs are not an essential part of the pattern generator for feeding. The data provide strong support for the idea that the MCCs do not function as command neurons since their presence is not necessary for the elicitation of feeding (Kupfermann and Weiss, 1978). Moreover, it has been shown in other experiments that firing of the MCCs is not sufficient to elicit feeding (Weiss et al., 1978, 1986b). The data are, however, consistent with the notion that the MCCs are modulatory neurons that alter ongoing motor activity. More specifically, the evidence suggests that the actions of the MCCs are, in fact, more limited. The MCCs may be selectively involved in biting responses as distinct from swallowing, a behavior in which a continuous strip or sheet of seaweed is ingested without radula protraction beyond the mouth of the animal. Furthermore, our analyses of biting responses of MCC-lesioned animals suggest that the rising phase of the bite cycle (consisting of forward rotation of the radula) is only modestly affected by lesioning, whereas the falling phase (consisting of backward rotation and radula closing) is markedly slowed. The falling phase may also be affected by a slowing of the relaxation of forward rotation. A deficit in backward rotation and radula closing is consistent with the known actions of the accessory radula closer (ARC), the buccal muscle whose MCC innervation has been most extensively studied (Cohen et al., 1978; Weiss et al., 1978, 1979, 1982). Unpublished observations (J. L. Cohen, E. C. 
Cropper, I. Kupfermann, and K. R. Weiss) suggest that other buccal muscles innervated by the MCCs are predominantly radula closers, retractors, or backward rotators.

It should be noted that although MCC lesioning resulted in a slowing of bite rate (tested when the animal was not permitted to ingest the food), the amount of food consumed in Experiment 2 and the time to reach satiation were not significantly affected by the lesioning. This seeming contradiction may be explained by 2 possible factors. First, during ingestion of the soft food we used, a substantial amount of time is taken to swallow the food, and our data suggest that swallow rate may not be affected by MCC lesioning (Fig. 14). Second, our method of feeding one piece at a time results in a meal in which there is "dead time" between bites and swallows, during which the experimenter prepares the new piece for feeding. Under these conditions the major contribution to the meal duration may consist of these dead times, which are under the control of the experimenter and are not subject to an effect of $\mathrm{MCC}$ lesioning.

\section{Effect of MCC lesioning on bite magnitude}

The relatively modest effects of $\mathrm{MCC}$ lesioning on the magnitude of repetitive biting responses may be the consequence of a variety of factors that could be acting separately or conjointly. Electrophysiological experiments have consistently shown that the force of buccal muscle contraction elicited by activation of buccal motoneurons innervating the muscle is enhanced by activity of the MCCs (Weiss et al., 1975, 1978). In the present experiments, the force of buccal muscle contraction was not measured directly. Our estimate of the extent of radula forward rotation only indirectly assesses the actual force of muscle contraction. Moreover, it is less likely to detect weakness of backward rotation, than of forward rotation. If one assumes that backward rotator and forward rotator muscles act as antagonists throughout the biting cycle, and further that the MCC lesioning preferentially weakens backward rotators, then one might expect exaggerated forward rotation during the forward rotation phase, followed by weakened or slowed backward rotation during the retraction phase. Furthermore, when the time for forward rotation is increased, because of a slowing of the cycle time, the radula will have an increased time to rotate forward, and hence might exhibit an increase in the bite magnitude measurement, even if the force of the movement was decreased. This mechanism might explain the small but anomalous increase in bite magnitude measured during the first few bites, when the bite cycle is particularly slowed in MCC-lesioned animals. It might also account for the exaggerated radula protraction ("stuck" radula) seen in 5 of $6 \mathrm{MCC}$-lesioned animals but in none of the control group animals. It is interesting that recent evidence indicates that 5-HT and other putative neuromodulators of buccal muscle not only enhance the magnitude of muscle contraction, but also increase the rate of relaxation of the muscle (Ram et al., 1984; Cropper et al., 1987b, 1988). Therefore, the prolongation of radula forward rotation in MCC-lesioned animals might be due, in part, to the absence of 5-HT release at buccal muscles, as well as at central neurons that determine the characteristics of the central pattern generator for biting.

A second possible explanation for the observed effects of $\mathrm{MCC}$ lesioning on bite magnitude is that under normal conditions during the course of a meal the effects of MCC activity on the muscle are cumulative and may contribute to an antifatiguing process that comes into play only after an animal has been feeding for a considerable period of time. Lesioned animals would therefore be expected to show a progressive deficit that would develop late in the meal as fatigue, or some related mechanism that depresses responding, comes into play (see Figs. 12, $B, C$ and 13). MCC activity may also counter fatigue during a series of closely spaced bites (see last 3 bites in Fig. 7). These observations suggest that MCC enhancement of consummatory responding may have both long- and short-term effects.

\section{Extrinsic and intrinsic modulation of feeding}

A provocative third possible explanation for the relatively small effect of MCC lesioning on bite magnitude is that the buccal motor system may be significantly modulated by other extrinsic modulatory neurons or by modulatory processes intrinsic to the motor system. Supporting this idea is the observation that MCClesioned animals show a surprising degree of modulation of the speed and magnitude of their biting responses, both during the course of the successive bites within a testing run (Figs. 7, 8), as well as over the course of a meal (Figs. 11B, 12B). For example, the mean IBIs of MCC-lesioned animals at the start of a testing run (Fig. 7) were more than 200\% longer than those of controls. During the subsequent 5 biting responses, the intervals fall gradually to approximately $130 \%$ of controls. Concomitantly, under our testing conditions, during the 5 initial biting responses of MCC-lesioned animals, mean bite magnitude increased approximately $13 \%$, which is virtually the same as the build-up of bite magnitude seen with control animals (Fig. 8). Indeed, we previously suggested that arousal may be, in part, an emergent property of the motor system and that an intrinsic property such as posttetanic potentiation (PTP) at the neuromuscular junction may contribute to the build-up of the force of contraction at the beginning of a series of buccal contractions (Cohen et al., 1978; Weiss et al., 1982). The current findings support the idea that intrinsic plastic properties, both peripheral and central, may contribute to the expression of arousal at the level of biting behavior. Recent data (Lloyd et al., 1984, 1987; Cropper et al., 1987a, b, 1988) suggest that the buccal motor system may possess an additional source of modulatory control over buccal muscle contractions. Buccal motoneurons (B15 and B16) for the ARC muscle contain neuropeptides that can potentiate muscle contraction and increase relaxation rate, in a manner similar to what is seen in response to $5-\mathrm{HT}$, the transmitter of the MCCs. Preoperatively, when the animals are highly motivated, the combined enhancement of muscle activity due to intrinsic plastic properties of the motor system (including PTP and the action of neuropeptides) and the extrinsic actions of the MCCs may be more than sufficient to achieve a maximal force of contraction, and hence maximal bite magnitude, i.e., a ceiling effect on bite magnitude may be reached. Following lesioning of the MCCs, the intrinsic plastic properties of the motor system alone may be sufficient to achieve maximal bite magnitudes (albeit at the expense of a slowing of the feeding program), providing that a high motivational state is maintained. However, when the motor system is depressed, for example, due to satiation or to general effects of fatigue, the contribution of the MCCs may assume increasing importance. Support for this interpretation comes from the finding that during the last meal deciles of complete meals, the mean bite magnitudes of the MCC-lesioned animals were below those of the controls (Figs. 12, 13). Additional support is suggested by the finding that toward the end of the sham feeding control meal, when fatigue could begin to be a factor affecting bite magnitude, the MCC Lesion group began to show a decline in bite magnitude 
that was not seen for the Control group (Fig. 12C). The magnitude of buccal muscle contraction is proportional to the size of the muscle junction potentials (Cohen et al., 1978), and it is therefore also possible that as animals satiate and IBI increases, posttetanic potentiation at the muscle contributes progressively less to the magnitude of contraction and that there is therefore a relative increase of the contribution of MCC activity. It remains for future work to determine how the central and peripheral neuromodulatory systems interact to control the expression of motivational states such as food-induced arousal and satiation in Aplysia.

\section{References}

Allison, P., and P. R. Benjamin (1986) Stimulation of neuritic outgrowth in an undamaged molluscan interneurone. J. Exp. Biol. 122: $447-451$.

Altrup, U., and G. Kolde (1982) Changes in neuronal arborizations induced by lesions of the ganglionic perineurium (Helix pomatia) Neurosci. Lett. 34: 215-220.

Barber, A. (1983) Properties of the serotonergic cerebral ganglion neurons of the gastropod mollusc, Philine aperta. Comp. Biochem. Physiol. $76 \mathrm{C}: 135-149$.

Bowling, D., J. Nicholls, and I. Parnas (1978) Destruction of a single cell in the central nervous system of the leech as a means of analysing its connexions and functional role. J. Physiol. (Lond.) 282: 169-180.

Bulloch, A. G. M., and S. B. Kater (1982) Neurite outgrowth and selection of new electrical connections by adult Helisoma neurons. J. Neurophysiol. 48: 569-583.

Chiel, H. J., K. R. Weiss, and I. Kupfermann (1986) An identified histaminergic neuron modulates feeding motor circuitry in Aplysia. J. Neurosci. 6: 2427-2450.

Chow, K. L. (1967) Effects of ablation. In The Neurosciences, G. L. Quarton, T. Melnechuk, and F. O. Schmitt, eds., pp. 705-713, Rockefeller U. P., New York.

Coggeshall, R. E. (1967) A light and electron microscope study of the abdominal ganglion of Aplysia californica. J. Neurophysiol. 30: $1263-$ 1287.

Cohen, J. L., K. R. Weiss, and I. Kupfermann (1978) Motor control of buccal muscles in Aplysia. J. Neurophysiol. 41: 157-180.

Comer, C. M. (1985) Analyzing cockroach escape behavior with lesions of individual giant interneurons. Brain Res. 335: 342-346.

Cropper, E. C., P. E. Lloyd, W. Reed, R. Tenenbaum, I. Kupfermann, and K. R. Weiss (1987a) Multiple neuropeptides in cholinergic motor neurons of Aplysia: Evidence for modulation intrinsic to the motor circuit. Proc. Natl. Acad. Sci. USA 84: 3486-3490.

Cropper, E. C., R. Tenenbaum, M. A. G. Kolks, I. Kupfermann, and K. R. Weiss (1987b) Myomodulin: A bioactive neuropeptide present in an identified cholinergic buccal motor neuron of Aplysia. Proc. Natl. Acad. Sci. USA 84: 5483-5486.

Cropper, E. C., M. W. Miller, R. Tenenbaum, M. A. G. Kolks, I. Kupfermann, and K. R. Weiss (1988) Structure and action of buccalin: A modulatory neuropeptide localized to an identified SCP-containing cholinergic motor neuron of Aplysia californica. Proc. Natl. Acad. Sci. USA 85: 6177-6181.

Eisenstadt, M., J. E. Goldman, E. R. Kandel, H. Koike, J. Koester, and J. H. Schwartz (1973) Intrasomatic injection of radioactive precursors for studying transmitter synthesis in identified neurons of Aplysia californica. Proc. Natl. Acad. Sci. USA 70: 3371-3375.

Gelperin, A. (1981) Synaptic modulation by identified serotonin neurons. In Serotonin Neurotransmission and Behavior, B. L. Jacobs and A. Gelperin, eds., pp. 288-304, MIT Press, Cambridge, MA.

Gerschenfeld, II. M., M. IIamon, and D. Paupardin-Tritsch (1978) Release of endogenous serotonin from two identified serotonin-containing neurones and the physiological role of serotonin reuptake. J. Physiol. (Lond.) 274: 265-278.

Gillette, R., and W. J. Davis (1977) The role of the metacerebral giant neuron in the feeding behavior of Pleurobranchaea. J. Comp. Physiol. 116: $129-159$.

Goldstein, R. S., K. R. Weiss, and J. H. Schwartz (1982) Intraneuronal injection of horseradish peroxidase labels glial cells associated with the axons of the giant metacerebral neuron of Aplysia. J. Neurosci. 2: $1567-1577$.
Granzow, B., and S. B. Kater (1977) Identified higher-order neurons controlling the feeding motor program of Helisoma. Neuroscience 2 . 1049-1063.

Hamilton, C. L. (1969) Problems of refeeding after starvation in the rat. Ann. NY Acad. Sci. 157: 1004-1017.

Jahan-Parwar, B., and S. M. Fredman (1976) Cerebral ganglion of Aplysia: Cellular organization and origin of nerves. Comp. Biochem. Physiol. 54A: 347-357.

Kupfermann, I. (1974a) Feeding behavior in Aplysia: A simple system for the study of motivation. Behav. Biol. 10:1-26.

Kupfermann, I. (1974b) Dissociation of the appetitive and consummatory phases of feeding in Aplysia: A lesion study. Behav. Biol. 10: 89-97.

Kupfermann, I., and K. R. Weiss (1978) The command neuron concept. Behav. Brain Sci. 1: 3-39.

Kupfermann, I., and K. R. Weiss (1981) The role of serotonin in arousal of feeding behavior of Aplysia. In Serotonin Neurotransmission and Behavior, B. L. Jacobs and A. Gelperin, eds., pp. 255-287, MIT Press, Cambridge, MA.

Kupfermann, I., and K. R. Weiss (1982) Activity of an identified serotonergic neuron in freemoving Aplysia correlates with behavioral arousal. Brain Res. 241: 334-337.

Kuslansky, B., K. R. Weiss, and I. Kupfermann (1987) Mechanisms underlying satiation of feeding behavior of the mollusc Aplysia. Behav. Neurol. Biol. 48: 278-303.

Lloyd, P. E., I. Kupfermann, and K. R. Weiss (1984) Evidence for parallel actions of a molluscan neuropeptide and serotonin in mediating arousal in Aplysia. Proc. Natl. Acad. Sci. 81: 2934-2937.

Lloyd, P. E., M. Frankfurt, P. Stevens, I. Kupfermann, and K. R. Weiss (1987) Biochemical and immunocytological localization of the neuropeptides FMRFamide, $\mathrm{SCP}_{A}, \mathrm{SCP}_{\mathrm{B}}$, to neurons involved in the regulation of feeding in Aplysia. J. Neurosci. 7: 1123-1132.

McCrohan, C. R., and T. E. Audesirk (1987) Initiation, maintenance and modification of patterned buccal motor output by the cerebral giant cells of Lymnaea stagnalis. Comp. Biochem. Physiol. 87 A:969977.

McCrohan, C. R., and P. R. Benjamin (1980) Synaptic relationships of the cerebral giant cells with motoneurones in the feeding system of Lymnaea stagnalis. J. Exp. Biol. 85: 169-186.

Murphy, A. D., D. L. Barker, J. F. Loring, and S. B. Kater (1985) Sprouting and functional regeneration of an identified serotonergic neuron following axotomy. J. Neurobiol. 16: 137-151.

Parnas, I., and D. Bowling (1977) Killing of single neurones by intracellular injection of proteolytic enzymes. Nature 270: 626-628.

Pentreath, V. W., M. S. Berry, and N. N. Osborne (1982) The serotonergic cerebral cells in gastropods. In Biology of Serotonergic Transmission, N. N. Osborne, ed., pp. 457-513, Wiley, New York.

Ram, J. L., G. S. Ajimal, D. Gole, K. A. Haller, and A. Williams (1984) Serotonin and forskolin enhance both magnitude of contraction and relaxation rate of Aplysia dorsal extrinsic muscle independently of acetylcholine receptor. Comp. Biochem. Physiol. 79C: 455-459.

Rosen, S. C., K. R. Weiss, J. L. Cohen, and I. Kupfermann (1982) Interganglionic cerebral-buccal mechanoafferents of Aplysia: Receptive fields and synaptic connections to different classes of neurons involved in feeding behavior. J. Neurophysiol. 48: 271-288.

Rosen, S. C., I, Kupfermann, R. S. Goldstein, and K. R. Weiss (1983) Lesion of a serotonergic modulatory neuron in Aplysia produces a specific defect in feeding behavior. Brain Res. 260: 151-155.

Rosen, S. C., M. W. Miller, K. R. Weiss, and I. Kupfermann (1987) Control of buccal motor programs in Aplysia by identified neurons in the cerebral ganglion. Soc. Neurosci. Abstr. 13: 1061.

Susswein, A. J., and J. H. Byrne (1988) Identification and characterization of neurons initiating patterned neural activity in the buccal ganglia of Aplysia. J. Neurosci. 8: 2049-2061.

Susswein, A. J., and I. Kupfermann (1975a) Bulk as a stimulus for satiation in Aplysia. Behav. Biol. 13: 203-209.

Susswein, A. J., and I. Kupfermann (1975b) Localization of bulk stimuli underlying satiation in Aplysia. J. Comp. Physiol. 101: 309328.

Susswein, A. J., I. Kupfermann, and K. R. Weiss (1976) The stimulus control of biting in Aplysia. J. Comp. Physiol. 108: 75-96.

Susswein, A. J., K. R. Weiss, and I. Kupfermann (1978) The effects of food arousal on the latency of biting in Aplysia. J. Comp. Physiol. 123: 31-41.

Thompson, E. B., J. H. Schwartz, and E. R. Kandel (1976) A radioau- 
tographic analysis in the light and electron microscope of identified Aplysia neurons and their processes after intrasomatic injection of L- $\left({ }^{3} \mathrm{H}\right)$ fucose. Brain Res. 112: 251-281.

Weinreich, D., M. W. McCaman, R. E. McCaman, and J. E. Vaughn (1973) Chemical, enzymatic and ultrastructural characterization of 5-hydroxytryptamine-containing neurons from the ganglia of Aplysia californica and Tritonia diomedia. J. Neurochem. 20: 969-976.

Weiss, K. R., and I. Kupfermann (1976) Homology of the giant serotonergic neurons (metacerebral cells) in Aplysia and pulmonate molluscs. Brain Res. 117: 33-49.

Weiss, K. R., and I. Kupfermann (1977) Serotonergic neuronal activity and arousal of feeding in Aplysia califurnica. In Society for Neuroscience Symposia, Vol. 3, Aspects of Behavioral Neurobiology, J. A. Ferrendelli, ed., pp. 66-89, Bethesda, MD.

Weiss, K. R., J. Cohen, and I. Kupfermann (1975) Potentiation of muscle contraction: A possible modulatory function of an identified serotonergic cell in Aplysia. Brain Res. 99: 381-386.

Weiss, K. R., J. L. Cohen, and I. Kupfermann (1978) Modulatory control of buccal musculature by a serotonergic neuron (metacerebral cell) in Aplysia. J. Neurophysiol. 41: 181-203.

Weiss, K. R., D. E. Mandelbaum, M. Schonberg, and I. Kupfermann (1979) Modulation of buccal muscle contractility by serotonergic metacerebral cells in Aplysia: Evidence for a role of cyclic adenosine monophosphate. J. Neurophysiol. 42: 791-803.

Weiss, K. R., U. T. Koch, J. Koester, S. C. Rosen, and I. Kupfermann (1982) The role of arousal in modulating feeding behavior of Aplysia: Ncural and bchavioral studies. In The Neural Basis of Feeding and Reward, B. G. Hoebel and D. Novin, eds., pp. 25-57, Haer Institute, Brunswick, ME

Weiss, K. R., E. Shapiro, and I. Kupfermann (1986a) Modulatory synaptic actions of an identified histaminergic neuron on the serotonergic metacerebral cell of Aplysia. J. Neurosci. 6: 2393-2402.

Weiss, K. R., H. J. Chiel, U. Koch, and I. Kupfermann (1986b) Activity of an identified histaminergic neuron, and its possible role in arousal of feeding in semi-intact Aplysia. J. Neurosci. 6: 2403-2415. 